\title{
On the quasi-sure superhedging duality with frictions
}

\author{
Erhan Bayraktar $^{1} \cdot$ Matteo Burzoni $^{2}$
}

Received: 30 May 2019 / Accepted: 16 September 2019 / Published online: 21 November 2019

(C) The Author(s) 2019

\begin{abstract}
We prove the superhedging duality for a discrete-time financial market with proportional transaction costs under model uncertainty. Frictions are modelled through solvency cones as in the original model of Kabanov (Finance Stoch. 3:237248,1999 ) adapted to the quasi-sure setup of Bouchard and Nutz (Ann. Appl. Probab. 25:823-859, 2015). Our approach allows removing the restrictive assumption of no arbitrage of the second kind considered in Bouchard et al. (Math. Finance 29:837$860,2019)$ and showing the duality under the more natural condition of strict no arbitrage. In addition, we extend the results to models with portfolio constraints.
\end{abstract}

Keywords Model uncertainty · Superhedging · Proportional transaction costs · Portfolio constraints $\cdot$ Robust finance

Mathematics Subject Classification (2010) 90C15 • 90C39 • 91G99 • 28A05 · 46A20

JEL Classification C61 - G13

Erhan Bayraktar is supported in part by the National Science Foundation under the grant DMS-1613170, and in part by the Susan M. Smith Chair. Matteo Burzoni gratefully acknowledges support by the ETH Foundation and the Hooke Research Fellowship from the University of Oxford.

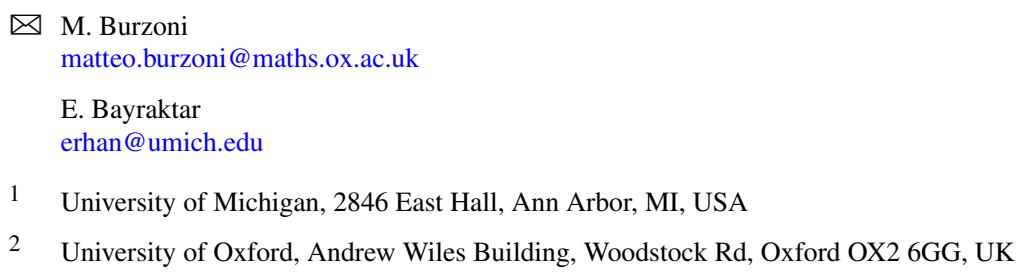




\section{Introduction}

It is more often the rule, rather than the exception, that socio-economic phenomena are influenced by a strong component of randomness. Starting from the pioneering work of Knight (see e.g. Knight [22, Chap. VII]), a distinction between risk and uncertainty has been widely accepted with respect to the nature of such a randomness. We often call a situation risky if a probabilistic description is available (e.g. the toss of a fair coin). In contrast, we call a situation uncertain if it cannot be fully described in probabilistic terms. Simple reasons could be the absence of an objective model (e.g. the result of a horse race; see Bayraktar and Munk [6] and the references therein) or the lack of information (e.g. the draw from an urn whose composition is unknown). The classical literature in mathematical finance has been mainly focusing on risk, and the attention to problems of Knightian uncertainty has been drawn only relatively recently starting from Avellaneda et al. [4]. In particular, fundamental topics such as the theory of arbitrage and the related superhedging duality have been systematically studied in frictionless discrete-time markets in Bayraktar and Zhou [8], Bouchard and Nutz [12] in a quasi-sure framework, and in Acciaio et al. [1], Burzoni et al. [15], Cheridito et al. [17] in a pointwise framework.

Under risk, the classical model of a discrete-time market with proportional transaction costs has been introduced in Kabanov [20]. The model is described by a collection of cones $\mathbb{K}:=\left(K_{t}\right)_{t=0, \ldots, T}$ which determines (i) admissible strategies, (ii) solvency requirements, (iii) pricing mechanisms. More precisely, the latter are called consistent price systems, and they are essentially martingale processes taking values in the dual cones $K_{t}^{*}$. Instances of such models have been considered, in the uncertainty case, in Bartl et al. [5], Bayraktar and Zhang [7], Bouchard and Nutz [13], Burzoni [14], Dolinsky and Soner [19]; nevertheless, the problem of establishing a quasi-sure superhedging duality has remained open. Recently, a first duality result was obtained in Bouchard et al. [11] using a randomisation approach (see also Aksamit et al. [2], Bayraktar and Zhou [9], Deng et al. [18] for other applications). The idea is to construct a fictitious frictionless price process $\hat{S}$ for which (i) the superhedging price of an option in the market with frictions coincides with the corresponding superhedging price in the frictionless one, and (ii) the class of martingale measures for $\hat{S}$ produces the same prices for the option as the class of consistent price systems for the original market. When these two properties are achieved, the duality follows from the frictionless results of Bouchard and Nutz [12]. In order to perform this program, a crucial role is played by the assumption of no arbitrage of the second kind $\left(\mathrm{NA}_{2}(\mathcal{P})\right)$, which ensures that the construction of the fictitious frictionless market is automatically arbitrage-free. $\mathrm{NA}_{2}(\mathcal{P})$ prescribes that if a position is quasisurely solvent at time $t+1$, it must be quasi-sure solvent at time $t$. Such a condition is quite restrictive as it fails in very basic examples of one-period markets, even though no sure profit can be made by market participants (see [7, Remark 11]).

In this paper, we do not require the strong assumption $\mathrm{NA}_{2}(\mathcal{P})$ and we show the superhedging duality under the more natural condition of strict no arbitrage $\left(\mathrm{NA}^{\mathrm{s}}(\mathcal{P})\right)$. The latter ensures that it is not possible to make profits without taking any risk; thus it generalises the classical no-arbitrage condition in frictionless markets. From a technical perspective, we also do not assume other unnecessary hypotheses 
taken in Bouchard et al. [11]: (i) we do not require that transaction costs are uniformly bounded, or stated differently, the bid-ask spreads relative to a chosen numéraire are not necessarily subsets of $[1 / c, c]$ for some $c>0$; (ii) we do not require the technical assumption $K_{t}^{*} \cap \partial \mathbb{R}_{+}^{d}=\{0\}$ for any $t=0, \ldots, T$. From a modelling perspective, our approach allows extending the previous results to models where a process of portfolio constraints $C:=\left(C_{t}\right)_{t=0, \ldots, T}$ defines the admissible strategies in the market. To the best of our knowledge, these results are new even in the classical case where a reference probability measure $\mathbb{P}$ is fixed. As in Bouchard et al. [11], we assume the so-called efficient friction hypothesis and adopt a randomisation approach.

We first construct a backward procedure similar to the one of Bayraktar and Zhang [7] and based on a dynamic programming approach (see also Burzoni and Šikić [16] for an extensive study of the related martingale selection problem). This procedure yields a new collection of cones $\tilde{\mathbb{K}}^{*}=\left(\tilde{K}_{t}^{*}\right)_{t=0, \ldots, T}$ which is in general different from the original $\mathbb{K}$ and is shown to be nonempty under the condition $\mathrm{NA}^{\mathrm{s}}(\mathcal{P})$. Notably, it is not possible to apply directly the results of Bouchard et al. [11] (or a straightforward adaptation of them) to $\tilde{\mathbb{K}}^{*}$. Indeed, in general, $\tilde{K}_{t}^{*}$ will only have an analytic graph as opposed to the Borel-measurability of $K_{t}^{*}$. The Borel-measurability assumption is crucial in order to apply the results of Bouchard and Nutz [12] in frictionless markets. To overcome this difficulty, we propose a new randomisation method. We do not design the frictionless process $\hat{S}$ to take values in $\tilde{\mathbb{K}}^{*}$, but instead consider a suitable class $\hat{\mathcal{P}}$ of probabilities in order to have $\hat{S}_{t} \in \tilde{K}_{t}^{*} \hat{\mathcal{P}}$-q.s. at each time. Similarly to Bouchard et al. [11], we finally prove that the desired duality can be deduced from duality results in frictionless market. In particular, we use here results of Bayraktar and Zhou [8], which take into account possible portfolio constraints.

We conclude the introduction by specifying the frequently used notation and the setup. The superhedging duality is stated in Sect. 2. The construction of the fictitious frictionless market is the content of Sect. 3. Finally, we prove the main result in Sect. 4 where we also show how it extends to semi-static trading.

Notation For a topological space $X, \mathcal{B}_{X}$ is the Borel sigma-algebra, $\mathfrak{P}(X)$ the class of all probability measures on $\left(X, \mathcal{B}_{X}\right)$ and $\delta_{x}$ the Dirac measure in $x \in X$. For a probability measure $\mathbb{P}$ and a set $\mathcal{R} \subseteq \mathfrak{P}(X)$, we say that $\mathbb{P} \ll \mathcal{R}$ if there exists $\tilde{\mathbb{P}} \in \mathcal{R}$ such that $\mathbb{P} \ll \tilde{\mathbb{P}}$. A property is said to hold $\mathcal{R}$-q.s. if it holds for any $\mathbb{P} \in \mathcal{R}$. A map $U$ defined on $X$ and taking values in the power set of a space $Y$ is called a multifunction and denoted by $U: X \rightrightarrows Y$. For $Y=\mathbb{R}^{d}$ and a sigma-algebra $\mathcal{G}$ on $X$, a multifunction $U$ is called $\mathcal{G}$-measurable if for any open set $O \subseteq \mathbb{R}^{d}$, we have

$$
\{x \in X: U(x) \cap O \neq \emptyset\} \in \mathcal{G} .
$$

A map $f: X \rightarrow \mathbb{R}^{d}$ with $f(x) \in U(x)$ for any $x \in \operatorname{dom} U:=\{x \in X: U(x) \neq \emptyset\}$ is called a selector of $U$. We denote by $\mathcal{L}^{0}(\mathcal{G} ; U)$ the class of $\mathcal{G}$-measurable selectors of $U$. For $U: X_{1} \times X_{2} \rightrightarrows \mathbb{R}^{d}$ and $x \in X_{1}$ fixed, the notation $U(x ; \cdot)$ refers to the multifunction $U$ viewed as a (multi)function on $X_{2}$. Given a class of probabilities $\mathcal{R} \subseteq \mathfrak{P}\left(X_{2}\right)$, the (conditional) quasi-sure support of $U(x ; \cdot)$, denoted by $\operatorname{supp}_{\mathcal{R}} U(x ; \cdot)$, is the smallest closed set $F \subseteq \mathbb{R}^{d}$ such that $U(x ; \cdot) \subseteq F \mathcal{R}$-q.s. For a collection of multifunctions $U:=\left(U_{t}\right)_{t=0}^{T}$ adapted to a given filtration $\mathbb{G}$, we denote by $\mathcal{L}^{0}\left(\mathbb{G}_{-} ; U\right)$ the class of processes $H$ such that $H_{t+1} \in \mathcal{L}^{0}\left(\mathcal{G}_{t} ; U_{t}\right)$ for ev- 
ery $t=0, \ldots, T-1$. Finally, for two $\mathbb{R}^{d}$-valued processes $H$ and $S$, we define $(H \bullet S)_{t}:=\sum_{u=0}^{t-1} H_{u+1} \cdot\left(S_{u+1}-S_{u}\right)$.

Setup Let $T \in \mathbb{N}$ be a fixed time horizon and $\mathcal{I}:=\{0, \ldots, T\}$. For later use, we also define $\mathcal{I}_{-1}:=\{-1, \ldots, T-1\}$. We consider a filtered space $\left(\Omega, \mathcal{F}, \mathcal{F}^{u}, \mathbb{F}, \mathbb{F}^{u}\right)$ endowed with a (possibly nondominated) class of priors $\mathcal{P} \subseteq \mathfrak{P}(\Omega)$ described as follows:

- $\Omega_{0}$ is a given Polish space, $\Omega:=\Omega_{T}$, where $\Omega_{t}$ denotes the $(t+1)$-fold product of $\Omega_{0}$. Any $\omega \in \Omega_{t}$ is denoted by $\omega=\left(\omega_{0}, \ldots, \omega_{t}\right)$ with $\omega_{s} \in \Omega_{0}$ for any $0 \leq s \leq t$.

- We set $\mathcal{F}:=\mathcal{B}_{\Omega}$ and call $\mathcal{F}^{u}$ its universal completion. Similarly, the filtrations $\mathbb{F}=\left(\mathcal{F}_{t}\right)_{t \in \mathcal{I}}$ and $\mathbb{F}^{u}=\left(\mathcal{F}_{t}^{u}\right)_{t \in \mathcal{I}}$ are given by $\mathcal{F}_{t}:=\mathcal{B}_{\Omega_{t}}$ and $\mathcal{F}_{t}^{u}$ is its universal completion.

- For each $t \in \mathcal{I}, \mathcal{P}_{t}$ is a multifunction on $\Omega_{t}$ with values in the power set of $\mathfrak{P}\left(\Omega_{0}\right)$ and analytic graphs, ${ }^{1}$ with $\mathcal{P}_{0}$ a constant multifunction. We set

$$
\mathcal{P}=\left\{P_{0} \otimes \cdots \otimes P_{T-1}: P_{t} \in \mathcal{L}^{0}\left(\mathcal{F}_{t}^{u} ; \mathcal{P}_{t}\right), \forall t \in\{0, \ldots, T-1\}\right\} .
$$

The class $\mathcal{L}^{0}\left(\mathcal{F}_{t}^{u} ; \mathcal{P}_{t}\right)$ is nonempty due to the Jankov-von Neumann theorem (see [10, Proposition 7.49]) so that $\mathcal{P}$ is well defined through Fubini's theorem.

\section{Main result}

We consider the general model of financial markets with proportional transaction costs introduced in Kabanov [20]. The model is fully described by a collection of multifunctions $\mathbb{K}:=\left(K_{t}\right)_{t \in \mathcal{I}}$ with values in the family of convex closed cones in $\mathbb{R}^{d}$ with $d \geq 2$, called solvency cones. These represent the sets of positions, in terms of physical units of $d$ underlying assets, which can be liquidated to the zero portfolio at zero cost. We assume that any position with nonnegative coordinates is solvent, i.e., $\mathbb{R}_{+}^{d} \subseteq K_{t}$. The set $-K_{t}$ represents the class of portfolios which are available at zero cost. We assume that $K_{t}$ is $\mathcal{F}_{t}$-measurable for any $t \in \mathcal{I}$ with $K_{0}$ nonrandom. Following standard notation, for a cone $K \subseteq \mathbb{R}^{d}$, we denote by $K^{*}:=\left\{x \in \mathbb{R}^{d}: x \cdot k \geq 0, \forall k \in K\right\}$ its dual cone and by $K^{\bullet}:=-K^{*}$ its polar cone.

We generalise the model of Kabanov [20] by introducing constraints on the admissible positions in the market. These are represented by a collection of multifunctions $C:=\left(C_{t}\right)_{t \in \mathcal{I}}$ with values in the family of convex closed cones in $\mathbb{R}^{d}$ such that every $C_{t}$ is $\mathcal{F}_{t}$-measurable. A zero-cost strategy $\eta:=\left(\eta_{t}\right)_{t \in \mathcal{I}}$ is said to be admissible if it satisfies $\eta_{t} \in A_{t}$ for any $t \in \mathcal{I}$, where

$$
A_{t}:=\left\{\xi \in \mathcal{L}^{0}\left(\mathcal{F}_{t}^{u} ; C_{t}\right): \xi=\sum_{s=0}^{t}-k_{s} \text { with } k_{s} \in K_{s} \mathcal{P} \text {-q.s., } 0 \leq s \leq t\right\} .
$$

In words, $\eta$ satisfies the constraints imposed by $\left(C_{t}\right)_{t \in \mathcal{I}}$ and it is obtained as the sum of portfolios which are available at zero cost. We denote by $\mathcal{H}^{K}$ the class of

\footnotetext{
${ }^{1}$ We refer to [10, Chap. 7.6] for a detailed study of analytic sets.
} 
admissible strategies and omit the dependence on $C$ as it will be fixed throughout the paper.

Assumption 2.1 We assume that $\operatorname{int}\left(K_{t}^{*}\right) \neq \varnothing$ for any $t \in \mathcal{I}$. Moreover, we assume that $C_{t} \subseteq C_{t+1}$ for any $t=0, \ldots, T-1$.

The first assumption is known as efficient friction hypothesis. The second means that it is allowed to not trade between two periods, which is obviously satisfied in the unconstrained case where $C_{t} \equiv \mathbb{R}^{d}$ for any $t \in \mathcal{I}$.

Definition 2.2 The strict no arbitrage condition $\mathrm{NA}^{\mathrm{s}}(\mathcal{P})$ holds if for all $t \in \mathcal{I}$, we have $A_{t} \cap \mathcal{L}^{0}\left(\mathcal{F}_{t}^{u} ; K_{t}\right)=\{0\}$.

This condition is the straightforward generalisation to the quasi-sure setting of the classical one (see e.g. Kabanov et al. [21] and the recent paper Kühn and Molitor [23] for a slightly weaker variant of this concept).

Definition 2.3 A couple $(Z, \mathbb{Q})$ with $\mathbb{Q} \ll \mathcal{P}$ is called a (strictly) consistent price system $(S C P S)$ if $Z_{t} \in \operatorname{int}\left(K_{t}^{*}\right) \mathbb{Q}$-a.s., $\forall t \in \mathcal{I}$, and $H \bullet Z$ is a local $\mathbb{Q}$-supermartingale for all $H \in \mathcal{L}^{0}\left(\mathbb{F}_{-}^{u} ; C\right)$.

The interpretation is that $(Z, \mathbb{Q})$ defines a frictionless arbitrage-free price process which is compatible with the model of transaction costs defined by $\left(K_{t}, C_{t}\right)_{t \in \mathcal{I}}$. We shortly denote by $\mathcal{S}$ the set of SCPSs and by $\mathcal{S}^{0}$ the class of normalised SCPSs, i.e., those satisfying $Z_{t}^{d}=1$ for any $t \in \mathcal{I}$.

We are now ready to state the main result of the paper. Let $G: \Omega \rightarrow \mathbb{R}^{d}$ be a Borelmeasurable random vector which represents the terminal payoff of an option in terms of physical units of the underlying assets. The superhedging price of $G$ is given by

$$
\pi_{\mathbb{K}}(G):=\inf \left\{y \in \mathbb{R}: \exists \eta \in \mathcal{H}^{K} \text { such that } y e_{d}+\eta_{T}-G \in K_{T} \mathcal{P} \text {-q.s. }\right\},
$$

where $e_{d}$ is the $d$ th vector of the canonical basis of $\mathbb{R}^{d}$.

Theorem 2.4 Assume $\mathrm{NA}^{\mathrm{s}}(\mathcal{P})$. For any Borel-measurable random vector $G$,

$$
\pi_{\mathbb{K}}(G)=\sup _{(Z, \mathbb{Q}) \in \mathcal{S}} \mathbb{E}_{\mathbb{Q}}\left[G \cdot Z_{T}\right]
$$

Moreover, the superhedging price is attained when $\pi_{\mathbb{K}}(G)<\infty$.

The proof of Theorem 2.4 is given in Sect. 4. The main difficulty is to establish the result when only dynamic trading is allowed. In Theorem 4.12 below, we extend the duality to the case where also buy-and-hold positions in a finite number of options are allowed. In the following, it will be more convenient to extend the original market with an extra unconstrained component. More precisely, we consider the market $\overline{\mathbb{K}}$ with $\bar{K}_{t}:=K_{t} \times \mathbb{R}_{+}$and $\bar{C}_{t}:=C_{t} \times \mathbb{R}$ for $t \in \mathcal{I}$, which also satisfies Assumption 2.1. It is easy to see that $\pi_{\mathbb{K}}(G)=\pi_{\overline{\mathbb{K}}}(\bar{G})$ with $\bar{G}=(G, 0)$. 
On the dual side, $i: \mathcal{S} \rightarrow \overline{\mathcal{S}}^{0}$ with $i(Z, \mathbb{Q})=\left(\left(Z_{T}, 1\right), \mathbb{Q}\right)$ is clearly a bijection and $\mathbb{E}_{\mathbb{Q}}\left[G \cdot Z_{T}\right]=\mathbb{E}_{\mathbb{Q}}\left[\bar{G} \cdot\left(Z_{T}, 1\right)\right]$.

Without loss of generality, we assume that $\left(K_{t}, C_{t}\right)$ have one unconstrained component for any $t \in \mathcal{I}$.

We adapt some results of Bayraktar and Zhang [7] to the case of portfolio constraints. These will be useful in the next sections. We construct a collection of multifunctions $\tilde{\mathbb{K}}:=\left(\tilde{K}_{t}\right)_{t \in \mathcal{I}}$ starting from the definition of their dual sets. We let $\tilde{K}_{T}^{*}:=K_{T}^{*}$ and by backward recursion define

$$
\tilde{K}_{t}^{*}:=K_{t}^{*} \cap\left(\overline{\operatorname{conv}\left(\Gamma_{t}\right)}+C_{t}^{*}\right), \quad t=T-1, \ldots, 0,
$$

where for fixed $\omega \in \Omega_{t}$, we set $\Gamma_{t}(\omega):=\operatorname{supp}_{\mathcal{P}_{t}(\omega)} \tilde{K}_{t+1}^{*}(\omega ; \cdot)$. The following are generalisations of Lemma 6 and Proposition 4 in Bayraktar and Zhang [7] to the present setting. The proofs are analogous and we postpone them to the Appendix.

Lemma $2.5 \tilde{K}_{t}^{*}$ has analytic graph for every $t \in \mathcal{I}$.

Proposition 2.6 If $\mathbb{K}$ satisfies Assumption 2.1 and $\mathrm{NA}^{\mathrm{s}}(\mathcal{P})$, the same holds for $\tilde{\mathbb{K}}$. In particular, $\operatorname{int}\left(\tilde{K}_{t}^{*}\right) \neq \emptyset \mathcal{P}$-q.s. for all $t \in \mathcal{I}$.

\section{The randomisation approach}

In this section, we construct an enlarged measurable space $\left(\hat{\Omega}, \hat{\mathcal{F}}, \hat{\mathcal{F}}^{u}, \hat{\mathbb{F}}, \hat{\mathbb{F}}^{u}\right)$ endowed with a suitable class $\hat{\mathcal{P}}$ of probabilities. On this space, we construct a price process $\hat{S}=\left(\hat{S}_{t}\right)_{t \in \mathcal{I}}$ which represents a frictionless financial market with the property that $\hat{S}_{t} \in \tilde{K}_{t}^{*} \hat{\mathcal{P}}$-q.s. for any $t \in \mathcal{I}$ (Corollary 3.5 below) and which is arbitrage-free (Proposition 3.9 below).

We choose $\hat{\Omega}_{0}:=\Omega_{0} \times \mathbb{R}^{d-1}$ and set $\hat{\Omega}=\hat{\Omega}_{T}$, where $\hat{\Omega}_{t}$ denotes the $(t+1)$-fold product of $\hat{\Omega}_{0}$. We endow $\hat{\Omega}$ with the filtration $\hat{\mathbb{F}}:=\left(\hat{\mathcal{F}}_{t}\right)_{t \in \mathcal{I}}$, where for every $t \in \mathcal{I}$, we set $\hat{\mathcal{F}}_{t}:=\mathcal{F}_{t} \otimes \mathcal{B}_{\mathbb{R}^{d-1}}$. We denote by $\hat{\mathbb{F}}^{u}$ the universal completion of $\hat{\mathbb{F}}$. Similarly, we set $\hat{\mathcal{F}}:=\mathcal{B}_{\hat{\Omega}}$ and $\hat{\mathcal{F}}^{u}$ is its universal completion. We shortly write $(\omega, \theta) \in \hat{\Omega}_{t}$ for an element of the form $\left(\omega_{0}, \ldots, \omega_{t}, \theta_{0}, \ldots, \theta_{t}\right)$ with $\omega_{s} \in \Omega_{0}$ and $\theta_{s} \in \mathbb{R}^{d-1}$ for any $s=0, \ldots, t$. The collection of constraints extends to $\hat{\Omega}$ in the obvious way. Since there is no source of confusion, we still denote them by $C=\left(C_{t}\right)_{t \in \mathcal{I}}$. We next construct the price process $\hat{S}$. Recall that for any $t \in \mathcal{I}, K_{t}$ is Borel-measurable and thus so is $K_{t}^{*}$. Moreover, $\operatorname{int}\left(K_{t}^{*}\right)$ is nonempty by Assumption 2.1. From [13, Lemma A.1], there exists $S_{t} \in \mathcal{L}^{0}\left(\mathcal{F}_{t} ; \operatorname{int}\left(K_{t}^{*}\right)\right)$. Since $K_{t}^{*} \subseteq \mathbb{R}_{+}^{d}$, we can normalise $S_{t}$ with respect to e.g. the last component, so that $S_{t}$ takes values in

$$
K_{t}^{*, 0}:=\left\{y \in K_{t}^{*}: y^{d}=1\right\}, \quad t \in \mathcal{I} .
$$

We define a Borel-measurable price process $\hat{S}$ as

$$
\hat{S}_{t}(\omega, \theta)=\left(S_{t}^{1}(\omega) \theta_{t}^{1}, \ldots, S_{t}^{d-1}(\omega) \theta_{t}^{d-1}, 1\right), \quad(\omega, \theta) \in \hat{\Omega}, t \in \mathcal{I},
$$


where the last component serves as a numéraire. The rest of the section is devoted to the construction of the desired set $\hat{\mathcal{P}}$ of probability measures. For every $t \in \mathcal{I}$, we define the multifunctions

$$
\Theta_{t}(\omega):=\left\{\theta \in \mathbb{R}^{d-1}: \hat{S}_{t}(\omega, \theta) \in \operatorname{int}\left(\tilde{K}_{t}^{*}(\omega)\right)\right\}, \quad \omega \in \Omega_{t} .
$$

Lemma 3.1 For every $t \in \mathcal{I}, \Theta_{t}$ has an analytic graph.

Proof In the proof, we repeatedly use the fact that the class of analytic sets is closed under countable unions and intersections and that the image of an analytic set under a Borel-measurable function is again analytic.

1) For any $t \in \mathcal{I}$, consider the multifunction $\tilde{K}_{t}^{*, d-1}:=\operatorname{proj}_{\mathbb{R}^{d-1}}\left(\tilde{K}_{t}^{*, 0}\right)$, where the projection is taken over the first $d-1$ coordinates and $\tilde{K}_{t}^{*, 0}$ is the analogue of (3.1) for $\tilde{\mathbb{K}}$. Observe that

$$
\operatorname{graph}\left(\tilde{K}_{t}^{*, d-1}\right)=\operatorname{proj}_{\Omega_{t} \times \mathbb{R}^{d-1}}\left(\operatorname{graph}\left(\tilde{K}_{t}^{*}\right) \cap\left(\Omega_{t} \times \mathbb{R}^{d-1} \times\{1\}\right)\right) .
$$

From Lemma 2.5, $\operatorname{graph}\left(\tilde{K}_{t}^{*}\right)$ is analytic and so is the intersection in (3.4). As the projection is a continuous map, we conclude that $\operatorname{graph}\left(\tilde{K}_{t}^{*, d-1}\right)$ is analytic.

2) We now show that the set

$$
A_{t}^{v}:=\left\{(\omega, \theta) \in \Omega_{t} \times \mathbb{R}^{d-1}: \theta_{t}^{i}=\frac{y^{i}}{S_{t}^{i}(\omega)}, y \in \tilde{K}_{t}^{*, d-1}(\omega)+v\right\}
$$

is analytic, for each fixed $v \in \mathbb{R}^{d-1}$ and $t \in \mathcal{I}$. This together with Lemma A.1 in the Appendix yields the claim, as $\operatorname{graph}\left(\Theta_{t}\right)$ is the intersection of countably many analytic sets of the form $A_{t}^{v}$.

Observe that the function $f: \Omega_{t} \times \mathbb{R}^{d-1} \times \Omega_{t} \times \mathbb{R}^{d-1} \rightarrow \Omega_{t} \times \mathbb{R}^{d-1}$ defined as

$$
f(\omega, y, \tilde{\omega}, s)= \begin{cases}\left(\omega, \frac{y^{1}}{s^{1}}, \ldots, \frac{y^{d-1}}{s^{d-1}}\right), & \omega=\tilde{\omega} \\ (\omega,-1, \ldots,-1), & \omega \neq \tilde{\omega}\end{cases}
$$

is Borel-measurable. Recalling that $S_{t}>0$ and $K_{t}^{*} \subseteq \mathbb{R}_{+}^{d}$, we have that

$$
A_{t}^{v}=f\left(\operatorname{graph}\left(\tilde{K}_{t}^{*, d-1}+v\right), \operatorname{graph}\left(\tilde{S}_{t}\right)\right) \cap\left(\Omega_{t} \times \mathbb{R}_{+}^{d-1}\right),
$$

where $\tilde{S}$ is the process given by the first $d-1$ components of $S$. Since $\tilde{S}$ is Borelmeasurable, $\operatorname{graph}\left(\tilde{S}_{t}\right)$ is a Borel set. Moreover, from part 1$), \operatorname{graph}\left(\tilde{K}_{t}^{*, d-1}+v\right)$ is an analytic set. As $f$ is Borel-measurable, we conclude that $A_{t}^{v}$ is analytic.

Corollary 3.2 For any $t \in \mathcal{I}$, the multifunction

$$
\delta_{\Theta_{t}}(\omega):=\left\{\delta_{\theta} \in \mathfrak{P}\left(\mathbb{R}^{d-1}\right): \theta \in \Theta_{t}(\omega)\right\}
$$

has analytic graph. 
Proof The graph of $\delta_{\Theta_{t}}$ is the image of the graph of $\Theta_{t}$ via the map $(\omega, \theta) \mapsto\left(\omega, \delta_{\theta}\right)$, which is an embedding (see [3, Theorem 15.8]). Since the image of an analytic set under a continuous function is again analytic, the claim follows.

For $t \in\{0, \ldots, T-1\}$ and $\omega \in \Omega_{t}$, we define the multifunctions

$$
\hat{\mathcal{P}}_{t}(\omega):=\left\{\hat{\mathbb{P}} \in \mathfrak{P}\left(\hat{\Omega}_{0}\right):\left.\hat{\mathbb{P}}\right|_{\Omega_{0}} \in \mathcal{P}_{t}(\omega), \hat{\mathbb{P}}\left[\operatorname{graph}\left(\Theta_{t+1}(\omega ; \cdot)\right)\right]=1\right\} .
$$

We extend the definition to $t=-1$ with $\hat{\mathcal{P}}_{-1}:=\left\{\hat{\mathbb{P}} \in \mathfrak{P}\left(\hat{\Omega}_{0}\right): \hat{\mathbb{P}}\left[\operatorname{graph}\left(\Theta_{0}\right)\right]=1\right\}$, which is a constant multifunction as $K_{0}$ itself is a constant multifunction.

Proposition 3.3 The multifunctions $\hat{\mathcal{P}}_{t}$ defined in (3.5) have analytic graphs.

Proof Fix $t \in \mathcal{I}_{-1}$. For ease of notation, define $A_{t+1}:=\operatorname{graph}\left(\Theta_{t+1}\right)$, which is analytic from Lemma 3.1. The function $\mathbf{1}_{A_{t+1}}: \Omega_{t} \times \hat{\Omega}_{0} \rightarrow \mathbb{R}$ is thus upper semianalytic. It is not difficult to show that the function $\phi: \Omega_{t} \times \mathfrak{P}\left(\hat{\Omega}_{0}\right) \rightarrow \mathbb{R}$ such that $\phi(\omega, \hat{\mathbb{P}})=\mathbb{E}_{\hat{\mathbb{P}}}\left[\mathbf{1}_{A_{t+1}}(\omega ; \cdot)\right]$ is upper semianalytic (see [12, proof of Lemma 4.10]). As a consequence, the set

$$
\left\{(\omega, \hat{\mathbb{P}}) \in \Omega_{t} \times \mathfrak{P}\left(\hat{\Omega}_{0}\right): \hat{\mathbb{P}}\left[\operatorname{graph}\left(\Theta_{t+1}(\omega ; \cdot)\right)\right]=1\right\}=\phi^{-1}([1, \infty))
$$

is analytic as $\phi$ is upper semianalytic. In particular, the claim follows for $t=-1$.

Let now $0 \leq t \leq T-1$. Recall that the map $\pi_{\Omega_{0}}: \mathfrak{P}\left(\hat{\Omega}_{0}\right) \rightarrow \mathfrak{P}\left(\Omega_{0}\right)$ which associates to every $\hat{\mathbb{P}} \in \mathfrak{P}\left(\hat{\Omega}_{0}\right)$ its marginal on $\Omega_{0}$ is Borel-measurable (see [3, Theorem 15.14]). Therefore, as in [11, proof of Lemma 2.12 (i)], the set

$$
\left\{(\omega, \hat{\mathbb{P}}) \in \Omega_{t} \times \mathfrak{P}\left(\hat{\Omega}_{0}\right):\left.\hat{\mathbb{P}}\right|_{\Omega_{0}} \in \mathcal{P}_{t}(\omega)\right\}
$$

is also analytic. To conclude, observe that $\operatorname{graph}\left(\hat{\mathcal{P}}_{t}\right)$ is the intersection of the two previous sets.

We now show that $\hat{\mathcal{P}}_{t}$ is nonempty on a sufficiently rich set of events.

Lemma 3.4 Assume $\mathrm{NA}^{\mathrm{s}}(\mathcal{P})$. The set $N_{t}:=\left\{\omega \in \Omega_{t}: \hat{\mathcal{P}}_{t}(\omega)=\emptyset\right\}$ is a universally measurable $\mathcal{P}$-polar set for any $t \in \mathcal{I}_{-1}$. In particular, the same holds for the set $N:=\bigcup_{t \in \mathcal{I}_{-1}} N_{t}$.

Proof Fix $t \in \mathcal{I}_{-1}$. For $t=-1$, there is nothing to show as $\Theta_{0}$ is constant in $\omega$ and nonempty from Proposition 2.6. Suppose $0 \leq t \leq T-1$. Let $D_{t+1}:=\operatorname{dom}\left(\Theta_{t+1}\right)$ which is analytic from Lemma 3.1. As in the proof of Proposition 3.3, the function $\phi: \Omega_{t} \times \mathfrak{P}\left(\Omega_{0}\right) \rightarrow \mathbb{R}$ such that $\phi(\omega, \mathbb{P})=\mathbb{E}_{\mathbb{P}}\left[\mathbf{1}_{D_{t+1}}(\omega ; \cdot)\right]$ is upper semianalytic. We deduce that the set

$$
B_{t}:=\left\{(\omega, \mathbb{P}) \in \Omega_{t} \times \mathfrak{P}\left(\Omega_{0}\right): \phi(\omega, \mathbb{P})=1\right\} \cap \operatorname{graph}\left(\mathcal{P}_{t}\right)
$$

is analytic, and thus so is its projection on $\Omega_{t}$. Define $N_{t}^{\prime}:=\left(\operatorname{proj}_{\Omega_{t}}\left(B_{t}\right)\right)^{c} \in \mathcal{F}^{u}$. We show that under $\mathrm{NA}^{\mathrm{s}}(\mathcal{P}), N_{t}^{\prime}$ is $\mathcal{P}$-polar. To see this, observe that

$$
D_{t+1}^{c}=\left\{\omega \in \Omega_{t+1}: \operatorname{int}\left(\tilde{K}_{t+1}^{*}(\omega)\right)=\emptyset\right\}
$$


is $\mathcal{P}$-polar from Proposition 2.6. Suppose that there exists $\mathbb{P} \in \mathcal{P}$ such that $\mathbb{P}\left[N_{t}^{\prime}\right]>0$ and denote by $\left(P_{t}\right)_{t \in \mathcal{I}}$ its disintegration. By definition of $B_{t}, P_{t}\left[\omega, D_{t+1}^{c}\right]>0$ for every $\omega \in N_{t}^{\prime}$; therefore the random variable

$$
\int_{\Omega_{0}} \mathbf{1}_{D_{t+1}^{c}}\left(\omega ; \omega^{\prime}\right) P_{t}\left[\omega ; d \omega^{\prime}\right], \quad \omega \in \Omega_{t},
$$

is strictly positive on $N_{t}^{\prime}$. Since $\mathbb{P}\left[N_{t}^{\prime}\right]>0$, integrating over $P_{0} \otimes P_{t-1}$ yields $\mathbb{P}\left[D_{t+1}^{c}\right]>0$. This is a contradiction since $D_{t+1}^{c}$ is $\mathcal{P}$-polar.

It remains to show that $N_{t}^{\prime}=N_{t}$. The inclusion " $\subseteq$ " follows from the definition of $B_{t}$. Take now an $\mathcal{F}_{t}^{u}$-measurable selector $P_{t}$ of $B_{t}$ and $\delta_{\theta_{t+1}} \in \mathcal{L}^{0}\left(\mathcal{F}_{t+1}^{u} ; \delta_{\Theta_{t+1}}\right)$, where $\delta_{\Theta_{t+1}}$ is defined in Corollary 3.2. Since $P_{t}\left[\omega, \operatorname{dom}\left(\Theta_{t+1}\right)\right]=1$ for any $\omega \in\left(N_{t}^{\prime}\right)^{c}$, we can extend $\delta_{\theta_{t+1}}$ arbitrarily on the complement of $\operatorname{dom}\left(\Theta_{t+1}\right)$, and with a slight abuse of notation, we still denote it by $\delta_{\theta_{t+1}}$. The product measure $P_{t} \otimes \delta_{\theta_{t+1}}$ belongs to $\hat{\mathcal{P}}_{t}(\omega)$ for any $\omega \in\left(N_{t}^{\prime}\right)^{c}$. This shows $\left(N_{t}^{\prime}\right)^{c} \subseteq\left(N_{t}\right)^{c}$ and the thesis follows.

Corollary 3.5 Assume $\mathrm{NA}^{\mathrm{s}}(\mathcal{P})$. For any $t \in \mathcal{I}_{-1}$, we have $\hat{S}_{t+1} \in \operatorname{int}\left(\tilde{K}_{t+1}^{*}\right) \hat{\mathcal{P}}$-q.s. and for any $(\omega, \theta) \in N_{t}^{c} \times \mathbb{R}^{d-1}$,

$$
\operatorname{supp}_{\hat{\mathcal{P}}_{t}(\omega)} \hat{S}_{t+1}(\omega, \theta ; \cdot)=\operatorname{supp}_{\mathcal{P}_{t}(\omega)} \tilde{K}_{t+1}^{*, 0}(\omega ; \cdot) .
$$

Proof This follows because $P_{t} \otimes \delta_{\theta_{t+1}}$ belongs to $\hat{\mathcal{P}}_{t}$ for any $\delta_{\theta_{t+1}} \in \mathcal{L}^{0}\left(\mathcal{F}_{t+1}^{u} ; \delta_{\Theta_{t+1}}\right)$ and $P_{t}$ is a measurable selector of $B_{t}$ in (3.6).

Corollary 3.5 shows that the role of the parameter $\theta$ for the price process $\hat{S}$ is to "span" the dual cones given by the backward recursion (2.1). We set

$$
\hat{\mathcal{P}}:=\left\{\hat{P}_{-1} \otimes \cdots \otimes \hat{P}_{T-1}: \hat{P}_{t} \in \mathcal{L}^{0}\left(\mathcal{F}_{t}^{u} ; \hat{\mathcal{P}}_{t}\right), \forall t \in \mathcal{I}_{-1}\right\} .
$$

This class is well defined and constructed via Fubini's theorem as done for $\mathcal{P}$. Indeed, from Lemma 3.4, the set $N_{t}$ is $\mathcal{P}$-polar; thus we can extend arbitrarily any $\hat{P}_{t}$ to a universally measurable kernel which, with a slight abuse of notation, we still denote by $\hat{P}_{t}$. By construction, we have that the probability of the set of trajectories taking values in the interior of $\tilde{K}_{t}^{*}$ is equal to 1 , i.e.,

$$
\hat{\mathbb{P}}\left[(\omega, \theta) \in \hat{\Omega}: \hat{S}_{t}(\omega, \theta) \in \operatorname{int}\left(\tilde{K}_{t}^{*}(\omega)\right), \forall t \in \mathcal{I}\right]=1, \quad \forall \hat{\mathbb{P}} \in \hat{\mathcal{P}} .
$$

We finally show that starting from a model $(K, \mathcal{P})$ satisfying $\mathrm{NA}^{\mathrm{s}}(\mathcal{P})$, the induced frictionless market $(\hat{S}, \hat{\mathcal{P}})$ satisfies the no arbitrage condition of Definition 3.7 below.

Definition 3.6 We call a process $H$ an admissible strategy if $H_{t+1} \in \mathcal{L}^{0}\left(\hat{\mathcal{F}}_{t}^{u} ; C_{t}\right)$ and the self-financing condition $\left(H_{t+1}-H_{t}\right) \cdot \hat{S}_{t}=0 \hat{\mathcal{P}}$-q.s. holds for $0 \leq t \leq T-1$. The class of admissible strategies is denoted by $\hat{\mathcal{H}}^{r}$.

Definition 3.7 The no arbitrage condition $\mathrm{NA}(\hat{\mathcal{P}})$ holds if $(H \bullet \hat{S})_{T} \geq 0 \hat{\mathcal{P}}$-q.s. implies $(H \bullet \hat{S})_{T}=0 \hat{\mathcal{P}}$-q.s. for any $H \in \hat{\mathcal{H}}^{r}$. 
In order to use the frictionless duality results of Bayraktar and Zhou [8], we need to verify Assumptions 3.1 and 5.1 from that paper. Note that the set $\mathcal{H}_{t}$, in the notation of [8], corresponds to the set of constraints $C_{t}$ considered here. Under $\operatorname{NA}^{\mathrm{s}}(\mathcal{P})$, Corollary 3.5 and Proposition 2.6 imply that

$$
\operatorname{span}\left(\operatorname{supp}_{\hat{\mathcal{P}}_{t}(\omega)}\left(\hat{S}_{t+1}(\omega ; \cdot)-\hat{S}_{t}(\omega)\right)\right)=\mathbb{R}^{d-1} \times\{0\} \quad \hat{\mathcal{P}} \text {-q.s. },
$$

where for a set $U \subseteq \mathbb{R}^{d}, \operatorname{span}(U)$ denotes its linear hull. We deduce that the sets $\mathcal{H}_{t}$, $\mathcal{H}_{t}(\hat{\mathcal{P}})$ and $\mathcal{C}_{\mathcal{H}_{t}}(\hat{\mathcal{P}})$ in [8] all coincide $\hat{\mathcal{P}}$-q.s. with the first $d-1$ components of the set $C_{t}$. Since $C_{t}$ is a convex closed cone, Assumptions 3.1 (i), (ii) and 5.1 (i) are met. By [8, Remark 5.2], it is sufficient to verify Assumption 5.1 (ii). In particular, we show that

$$
A_{t}(\hat{\omega}, \hat{\mathbb{P}}):=\sup _{x \in C_{t}(\hat{\omega})}\left(x \cdot \mathbb{E}_{\hat{\mathbb{P}}}\left[\Delta \hat{S}_{t}(\hat{\omega} ; \cdot)\right]\right), \quad \hat{\omega} \in \hat{\Omega}_{t}, \hat{\mathbb{P}} \in \mathfrak{P}\left(\hat{\Omega}_{0}\right),
$$

is Borel-measurable. To see this, observe that $D:=\left\{(\hat{\omega}, \hat{\mathbb{P}}): \mathbb{E}_{\hat{\mathbb{P}}}\left|\Delta \hat{S}_{t}(\hat{\omega} ; \cdot)\right|<\infty\right\}$ is Borel-measurable as $\hat{S}$ is Borel-measurable (see e.g. [12, proof of Lemma 4.10]). Moreover, the function $F((\hat{\omega}, \hat{\mathbb{P}}), x):=x \cdot \mathbb{E}_{\hat{\mathbb{P}}}\left[\Delta \hat{S}_{t}(\hat{\omega} ; \cdot)\right]$ is a Carathéodory map, i.e., it is continuous in $x$ when $(\hat{\omega}, \hat{\mathbb{P}})$ are fixed and measurable in $(\hat{\omega}, \hat{\mathbb{P}})$ when $x$ is fixed. From [24, Example 14.15], the multifunction $F\left((\hat{\omega}, \hat{\mathbb{P}}), C_{t}(\hat{\omega})\right)$ is again Borelmeasurable. Finally, $A_{t}$ restricted to $D$ is again Borel-measurable since for any $c \in \mathbb{R}$,

$$
A_{t}^{-1}((c, \infty]) \cap D=\left\{(\hat{\omega}, \hat{\mathbb{P}}): F\left((\hat{\omega}, \hat{\mathbb{P}}), C_{t}(\hat{\omega})\right) \cap(c, \infty) \neq \emptyset\right\} \cap D .
$$

Let

$$
\hat{\mathcal{Q}}:=\left\{\mathbb{Q} \ll \hat{\mathcal{P}}: H \bullet \hat{S} \text { is a local } \mathbb{Q} \text {-supermartingale, } \forall H \in \mathcal{L}\left(\hat{\mathbb{F}}_{-}^{u} ; C\right)\right\} .
$$

The following is Theorem 3.2 of Bayraktar and Zhou [8], which is also valid in our context. For $\omega \in \Omega_{t}$ fixed, $\mathrm{NA}\left(\hat{\mathcal{P}}_{t}(\omega)\right)$ corresponds to $\mathrm{NA}(\hat{\mathcal{P}})$ for the one-period market $\left(\hat{S}_{t}(\omega), \hat{S}_{t+1}(\omega ; \cdot)\right)$.

Theorem 3.8 The following are equivalent:

(1) $\operatorname{NA}(\hat{\mathcal{P}})$.

(2) For any $0 \leq t \leq T-1, N_{t}^{\prime}:=\left\{(\omega, \theta) \in \hat{\Omega}_{t}: \operatorname{NA}\left(\hat{\mathcal{P}}_{t}(\omega)\right)\right.$ fails $\} \in \mathcal{F}^{u}$ is a $\hat{\mathcal{P}}$-polar set.

(3) For any $\mathbb{P} \in \hat{\mathcal{P}}$, there exists $\mathbb{Q} \in \hat{\mathcal{Q}}$ such that $\mathbb{P} \ll \mathbb{Q}$.

Proof The only difference from the proof of [8, Theorem 3.2] is that $\hat{\mathcal{P}}_{t}(\omega)$ might have empty values on the $\mathcal{P}$-polar set $N_{t} \in \mathcal{F}^{u}$. Recall that $\operatorname{graph}\left(\hat{\mathcal{P}}_{t}\right)$ is analytic by Proposition 3.3. Thus also $\operatorname{dom}\left(\hat{\mathcal{P}}_{t}\right)$ is an analytic set.

" $(1) \Rightarrow(2)$ " is proved in [8, Lemma 3.3]. It is shown there that $\left(N_{t}^{\prime}\right)^{c}$ is equal to the set $\left\{\omega \in \Omega_{t}:\left(\Lambda^{*} \cap C_{t}\right)(\omega) \subseteq-\Lambda^{*}(\omega)\right\}$, where we define the mapping $\Lambda$ by $\Lambda(\omega)=\operatorname{supp}_{\hat{\mathcal{P}}_{t}(\omega)}\left(\hat{S}_{t+1}(\omega ; \cdot)-\hat{S}_{t}(\omega)\right)$. In our framework, the above set must be intersected with $\operatorname{dom}\left(\hat{\mathcal{P}}_{t}\right)$ which is analytic, and therefore the intersection is again universally measurable. The same proof yields that $N_{t}^{\prime}$ is $\mathcal{P}$-polar. 
“(2) $\Rightarrow(3)$ " is based on [8, Lemma 3.4]. The universally measurable kernels $Q_{t}$ defining $\mathbb{Q} \in \hat{\mathcal{Q}}$ are constructed outside a $\mathcal{P}$-polar set, and in particular, they are chosen as selectors of a set $\Xi$ with $\operatorname{dom}(\Xi)=\left(N_{t}^{\prime}\right)^{c}$. In our framework, the same $\Xi$ satisfies $\operatorname{dom}(\Xi)=\left(N_{t} \cup N_{t}^{\prime}\right)^{c}$, which is still universally measurable and $\mathcal{P}$-polar. The same proof allows us to conclude.

" $(3) \Rightarrow(1)$ " is standard.

\section{Proposition 3.9 $\mathrm{NA}^{\mathrm{s}}(\mathcal{P})$ implies $\mathrm{NA}(\hat{\mathcal{P}})$.}

Proof Fix $t \in\{0, \ldots, T-1\}$. From Theorem 3.8, we only need to show that $\mathrm{NA}^{\mathrm{s}}(\mathcal{P})$ implies that the set $N_{t}^{\prime}$ is $\hat{\mathcal{P}}$-polar. Suppose there exists $H \in \mathcal{L}^{0}\left(\hat{\mathcal{F}}_{t}^{u} ; C_{t}\right)$ with

$$
H(\hat{\omega}) \cdot\left(\hat{S}_{t+1}(\hat{\omega} ; \cdot)-\hat{S}_{t}(\hat{\omega})\right) \geq 0 \quad \hat{\mathcal{P}}_{t}(\omega) \text {-q.s. }
$$

Corollary 3.5 implies that $H(\hat{\omega})$ (weakly) separates the singleton $\left\{\hat{S}_{t}(\hat{\omega})\right\}$ from the set $\operatorname{supp}_{\mathcal{P}_{t}(\omega)} \tilde{K}_{t+1}^{*}(\omega ; \cdot)$ for any $\hat{\omega}$ in the complement of a $\hat{\mathcal{P}}$-polar set. This separation extends to the closed convex hull $A_{t}(\hat{\omega}):=\overline{\operatorname{conv}\left(\Gamma_{t}(\omega)\right)}$, with the notation of (2.1). We can thus rewrite $H(\hat{\omega}) \in\left(A_{t}-\hat{S}_{t}\right)^{*}(\hat{\omega})$. Moreover, from the condition $H(\hat{\omega}) \in C_{t}(\hat{\omega})=C_{t}^{* *}(\hat{\omega})$, we also have $H(\hat{\omega}) \in\left(A_{t}+C_{t}^{*}-\hat{S}_{t}\right)^{*}(\hat{\omega})$. Finally, $(2.1)$ implies that $\operatorname{int}\left(\tilde{K}_{t}^{*}\right) \subseteq \operatorname{int}\left(A_{t}+C_{t}^{*}\right)$. We deduce that

$$
H(\hat{\omega}) \neq 0 \quad \Longrightarrow \quad \hat{S}_{t}(\hat{\omega}) \notin \operatorname{int}\left(\tilde{K}_{t}^{*}(\omega)\right) .
$$

By Corollary 3.5, $\hat{S}_{t} \in \operatorname{int}\left(\tilde{K}_{t}^{*}\right) \hat{\mathcal{P}}$-q.s.; thus $\left\{\hat{\omega} \in \hat{\Omega}_{t}: H(\hat{\omega}) \neq 0\right\}$ is $\hat{\mathcal{P}}$-polar.

\section{The superhedging duality}

This section is devoted to the proof of Theorem 2.4. To this end, we compare both the primal and the dual problem with their randomised counterpart in the frictionless market induced by $\hat{S}$ and constructed in Sect. 3. Using duality results known for the frictionless case, we obtain the result.

\subsection{Equality of the primal problems}

We first observe that using admissible strategies with respect to $\mathbb{K}$ or with respect to $\tilde{\mathbb{K}}$ yields the same superhedging price.

Lemma $4.1 \pi_{\mathbb{K}}(G)=\pi_{\tilde{\mathbb{K}}}(G)$.

Proof Since $K_{t} \subseteq \tilde{K}_{t}$, the inequality " $\geq$ " is trivial. Let now $(y, \tilde{\eta}) \in \mathbb{R} \times \mathcal{H}^{\tilde{K}}$ be a superhedge for $G$. We show that there exists $\eta \in \mathcal{H}^{K}$ such that $\eta_{T}=\tilde{\eta}_{T}$, and thus $(y, \eta)$ is a superhedge for $G$. By definition, we can write $\tilde{\eta}_{T}=\sum_{t=0}^{T}-\tilde{k}_{t}$ for some $\tilde{k}_{t} \in \mathcal{L}^{0}\left(\mathcal{F}_{t}^{u} ; \tilde{K}_{t}\right)$ for any $t \in \mathcal{I}$. We observe that from (2.1),

$$
\tilde{K}_{t}=\tilde{K}_{t}^{* *}=\left(K_{t}^{*} \cap\left(\overline{\operatorname{conv}\left(\Gamma_{t}\right)}+C_{t}^{*}\right)\right)^{*}=K_{t}+\left(\Gamma_{t}^{*} \cap C_{t}\right) .
$$


From [7, Lemma 8], $\tilde{k}_{t}=f+g$ with $f \in \mathcal{L}^{0}\left(\mathcal{F}_{t}^{u} ; K_{t}\right)$ and $g \in \mathcal{L}^{0}\left(\mathcal{F}_{t}^{u} ; \tilde{K}_{t+1} \cap C_{t}\right)$. Iterating the same procedure up to time $T-1$ and recalling that $\tilde{K}_{T}=K_{T}$, we obtain that

$$
\tilde{k}_{t}=f_{t}^{t}+\cdots+f_{T}^{t} \quad \text { for some } f_{s}^{t} \in \mathcal{L}^{0}\left(\mathcal{F}_{t}^{u} ; K_{s}\right), \forall s \in\{t, \ldots, T\}
$$

Moreover, $g_{s}^{t}:=\sum_{u=s+1}^{T} f_{u}^{t}$ belongs to $\mathcal{L}^{0}\left(\mathcal{F}_{t}^{u} ; \tilde{K}_{s+1} \cap C_{s}\right)$ for $s=t, \ldots, T-1$. Note that $f_{s}^{t}$ is defined only for $s \geq t$. We set $f_{s}^{t}=0$ for $s<t$ so that we can rewrite $\tilde{k}_{t}=\sum_{s=0}^{T} f_{s}^{t}$. that

Define now $k_{t}:=\sum_{s=0}^{t} f_{t}^{s}$ and $\eta_{t}:=\sum_{u=0}^{t}-k_{u}$ for $t \in \mathcal{I}$. Clearly, $\eta_{T}=\tilde{\eta}_{T}$ so

$$
y e_{d}+\tilde{\eta}_{T}-G \in K_{T} \quad \Longrightarrow \quad y e_{d}+\eta_{T}-G \in K_{T} .
$$

We are only left to show that $\eta_{t} \in \mathcal{L}^{0}\left(\mathcal{F}_{t}^{u} ; C_{t}\right)$ for any $t \in \mathcal{I}$. To this end, observe that for $t=T$, this follows from $\eta_{T}=\tilde{\eta}_{T}$. For $t=0, \ldots, T-1$, we have

$$
\sum_{u=0}^{t} \tilde{k}_{u}=\sum_{u=0}^{t}\left(\sum_{s=0}^{t} f_{s}^{u}+\sum_{s=t+1}^{T} f_{s}^{u}\right)=\sum_{s=0}^{t}\left(k_{s}+g_{t}^{s}\right),
$$

where for the second equality, we exchanged the order of summation in the first term and used the definition of $g_{t}^{s}$ in the second term. The above equation reads $\eta_{t}=\tilde{\eta}_{t}+\sum_{s=0}^{t} g_{t}^{s}$. By construction, $g_{t}^{s} \in C_{t} \mathcal{P}$-q.s. Moreover, the admissibility of $\tilde{\eta}$ implies that $\tilde{\eta}_{t} \in C_{t} \mathcal{P}$-q.s. By recalling that $C_{t}$ is a convex cone, the claim follows.

We now consider the superhedging problem in the frictionless market defined by $\hat{S}$. Note that a trading strategy in $\hat{\mathcal{H}}^{r}$ (see Definition 3.6) could in principle depend on the variable $\theta$. As this variable is only fictitious, a generic $\hat{\mathbb{F}}^{u}$-predictable process cannot consistently identify an element in $\mathcal{H}^{K}$. Therefore we need to reduce the class of admissible strategies to those which only depend on the variable $\omega$.

Definition 4.2 A consistent strategy $H=\left(H_{t}\right)_{t \in \mathcal{I}}$ is an $\mathbb{R}^{d}$-valued process satisfying $H_{t+1} \in \mathcal{L}^{0}\left(\mathcal{F}_{t}^{u} \otimes\left\{\varnothing, \mathbb{R}^{d}\right\} ; C_{t}\right)$ for any $0 \leq t \leq T-1$ and the self-financing condition

$$
\sup _{\theta \in \Theta_{t}}\left(H_{t+1}-H_{t}\right) \cdot \hat{S}_{t}(\cdot, \theta)=0 \quad \mathcal{P} \text {-q.s., } 0 \leq t \leq T-1 .
$$

We denote by $\hat{\mathcal{H}}$ the set of all self-financing consistent strategies.

Remark 4.3 Recall that the last component of $\hat{S}$ serves as a numéraire. The selffinancing condition for $H \in \hat{\mathcal{H}}^{r}$ is standard, namely, it requires that $-\left(H_{t+1}^{d}-H_{t}^{d}\right)$ coincides with $\sum_{i=1}^{d-1}\left(H_{t+1}^{i}-H_{t}^{i}\right) \hat{S}_{t}^{i} \hat{\mathcal{P}}$-q.s. On the other hand, a consistent strategy depends only on the $\omega$-variable and hence the position in the numéraire needs to be able to cover the worst-case scenario for the price of $\hat{S}_{t}$, which explains (4.1). We show below that for any consistent strategy, the left-hand side of (4.1) is measurable. 
Depending on the choice of admissible strategies, two corresponding superhedging prices of a random variable $g$ can be computed in the enlarged market, namely

$$
\begin{aligned}
\hat{\pi}^{r}(g) & :=\inf \left\{y \in \mathbb{R}: \exists H \in \hat{\mathcal{H}}^{r} \text { such that } y+(H \bullet \hat{S})_{T} \geq g \hat{\mathcal{P}} \text {-q.s. }\right\}, \\
\hat{\pi}(g) & :=\inf \left\{y \in \mathbb{R}: \exists H \in \hat{\mathcal{H}} \text { such that } y+(H \bullet \hat{S})_{T} \geq g \hat{\mathcal{P}} \text {-q.s. }\right\} .
\end{aligned}
$$

We want to show that the superhedging price of $G$ is equal to the superhedging price of $G \cdot \hat{S}_{T}$ in the frictionless market, using only consistent strategies. Towards this end, let us first elaborate on the self-financing condition for consistent strategies. For any $0 \leq t \leq T-1$, let $\Delta H_{t}:=H_{t+1}-H_{t}$ and define $F(\omega, x):=\sum_{i=1}^{d-1} \Delta H_{t}^{i}(\omega) x^{i}$, which is a Carathéodory map. Recall that the set $\tilde{K}_{t}^{*, d-1}$ from (1) in the proof of Lemma 3.1 has analytic graph and thus is universally measurable (see e.g. [7, Lemma 12]). From [24, Example 14.15], the multifunction $F\left(\omega, \tilde{K}_{t}^{*, d-1}\right)$ is again $\mathcal{F}_{t}^{u}$-measurable. We define $\phi_{t}(\omega):=\sup F\left(\omega, \tilde{K}_{t}^{*, d-1}(\omega)\right)$. Observe that $\phi_{t}$ is $\mathcal{F}_{t}^{u}$-measurable; indeed, for any $c \in \mathbb{R}$,

$$
\phi_{t}^{-1}((c, \infty])=\left\{\omega \in \Omega_{t}: F\left(\omega, \tilde{K}_{t}^{*, d-1}(\omega)\right) \cap(c, \infty) \neq \emptyset\right\} \in \mathcal{F}_{t}^{u}
$$

and $\phi_{t}^{-1}(\{\infty\})=\bigcap_{c \in \mathbb{Q}} \phi_{t}^{-1}((c, \infty]) \in \mathcal{F}_{t}^{u}$. We also observe that the self-financing condition (4.1) can be rewritten as

$$
-\Delta H_{t}^{d}=\sup _{\theta \in \Theta_{t}} \sum_{i=1}^{d-1} \Delta H_{t}^{i} \hat{S}_{t}^{i}(\cdot, \theta)=\sup _{x \in \tilde{K}_{t}^{*, d-1}} \sum_{i=1}^{d-1} \Delta H_{t}^{i} x^{i}=\phi_{t},
$$

where the second equality follows from Corollary 3.5. Set $A^{\infty}:=\bigcup_{t=0}^{T-1} \phi_{t}^{-1}(\{\infty\})$.

Lemma 4.4 Let $\mathbb{P} \in \mathcal{P}, H \in \hat{\mathcal{H}}$.

(1) Suppose that $\mathbb{P}\left[A^{\infty}\right]>0$. Then for any $n \in \mathbb{N}$, there exists $\hat{\mathbb{P}}^{n} \in \hat{\mathcal{P}}$ such that $\left.\hat{\mathbb{P}}^{n}\right|_{\Omega}=\mathbb{P}$ and $\hat{\mathbb{P}}^{n}\left[\sum_{i=1}^{d-1} \Delta H_{t}^{i} \hat{S}_{t}^{i} \geq n\right]>0$ for some $0 \leq t \leq T-1$.

(2) Suppose that $\mathbb{P}\left[A^{\infty}\right]=0$. Then for any $n \in \mathbb{N}$, there exists $\hat{\mathbb{P}}^{n} \in \hat{\mathcal{P}}$ such that $\left.\hat{\mathbb{P}}^{n}\right|_{\Omega}=\mathbb{P}$ and $\sum_{i=1}^{d-1} \Delta H_{t}^{i} \hat{S}_{t}^{i} \geq-\Delta H_{t}^{d}-\frac{1}{n} \hat{\mathbb{P}}^{n}-$ a.s. for any $0 \leq t \leq T-1$.

Proof For a fixed $\mathbb{P}$, we take a Borel-measurable version of $H$ (and therefore of $\phi_{t}$ ). We define the Carathéodory map $\hat{F}(\omega, \theta):=F\left(\omega, \hat{S}_{t}(\omega, \theta)\right)$ with $0 \leq t \leq T-1$ and $F$ as above, but with the difference that now $F$ is Borel-measurable in $\omega$. From [24, Example 14.15 (b)], the multifunctions

$$
\omega \mapsto\left\{\theta \times \mathbb{R}^{d-1}: \hat{F}(\omega, \theta) \geq n\right\}, \quad \omega \mapsto\left\{\theta \in \mathbb{R}^{d-1}: \hat{F}(\omega, \theta) \geq \phi_{t}(\omega)-\frac{1}{n}\right\}
$$

are Borel-measurable and therefore have Borel-measurable graphs. By intersecting their graphs with graph $\left(\Theta_{t}\right)$, we obtain two analytic sets. The Jankov-von Neumann theorem provides the existence of universally measurable selectors $\bar{\theta}_{t}^{\infty}$ and $\bar{\theta}_{t}^{<\infty}$, respectively. 
If $\mathbb{P}\left[A_{t}^{\infty}\right]>0$ for some $0 \leq t \leq T-1$, we let $\bar{\theta}_{t}=\bar{\theta}_{t}^{\infty}$ and for $s \neq t$, we let $\bar{\theta}_{s}$ be an arbitrary selector of $\Theta_{s}$. If $\mathbb{P}\left[A^{\infty}\right]=0$, we take $\bar{\theta}_{t}=\bar{\theta}_{t}^{<\infty}$ for any $0 \leq t \leq T-1$ and $\bar{\theta}_{T}$ an arbitrary selector of $\Theta_{T}$. Since $\mathbb{P}$ is fixed, we take Borel-measurable versions of the above selectors. In both cases, recalling that the map $\theta \mapsto \delta_{\theta}$ is an embedding of $\mathbb{R}^{d}$ into $\mathfrak{P}\left(\mathbb{R}^{d}\right)$, we construct a probability measure $\hat{\mathbb{P}}^{n}$ from the collection of kernels

$$
\hat{P}_{t}\left(\omega_{0}, \ldots, \omega_{t} ; \omega^{\prime}, \theta^{\prime}\right):=P_{t}\left[\omega_{0}, \ldots, \omega_{t} ; d \omega^{\prime}\right] \otimes \delta_{\bar{\theta}_{t+1}\left(\omega_{0}, \ldots, \omega_{t} ; \omega^{\prime}\right)}\left(\theta^{\prime}\right),
$$

for $0 \leq t \leq T-1$ and extend it to $\hat{P}_{-1}:=P_{-1} \otimes \delta_{\bar{\theta}_{0}}$ for an arbitrary $P_{-1} \in \mathfrak{P}\left(\Omega_{0}\right)$. The constructed $\hat{\mathbb{P}}^{n}$ satisfies $\left.\hat{\mathbb{P}}^{n}\right|_{\Omega}=\mathbb{P}$ and the desired properties.

$\operatorname{Lemma} 4.5 \pi_{\tilde{\mathbb{K}}}(G)=\hat{\pi}\left(G \cdot \hat{S}_{T}\right)$.

Proof " $\geq$ ": If the set of superhedging strategies for $G$ is empty, then $\pi_{\tilde{\mathbb{K}}}(G)=\infty$ and the inequality holds trivially. Suppose that $(y, \eta) \in \mathbb{R} \times \mathcal{H}^{\tilde{K}}$ is a superhedge for $G$. Define $H_{t+1}:=\eta_{t}$ for $t=0, \ldots, T-1$. Since $\tilde{K}_{T}$ is a convex cone and $\eta_{T}-\eta_{T-1} \in-\tilde{K}_{T}$ by admissibility, we have that

$$
y e_{d}+\eta_{T}-G \in \tilde{K}_{T} \Longrightarrow y e_{d}+\eta_{T-1}-G \in \tilde{K}_{T} .
$$

For any $\omega$ outside a $\mathcal{P}$-polar set and for any $s_{t} \in \tilde{K}_{t}^{*, 0}(\omega)$,

$$
\begin{aligned}
0 & \leq\left(y e_{d}+\eta_{T-1}(\omega)-G(\omega)\right) \cdot s_{T} \\
& \leq y+H_{T}(\omega) \cdot s_{T}+\sum_{t=0}^{T-1} k_{t}(\omega) \cdot s_{t}-G(\omega) \cdot s_{T} \\
& =y+\sum_{t=0}^{T-1} H_{t+1}(\omega) \cdot\left(s_{t+1}-s_{t}\right)-G(\omega) \cdot s_{T},
\end{aligned}
$$

where the second inequality follows from $k_{t} \in \tilde{K}_{t} \mathcal{P}$-q.s. for any $t \in \mathcal{I}$. Recalling that from (3.7), we have $\hat{S}_{t} \in \tilde{K}_{t}^{*} \hat{\mathcal{P}}$-q.s., we get $y+(H \bullet \hat{S})_{T} \geq G \cdot \hat{S}_{T} \hat{\mathcal{P}}$-q.s. It remains to show that, without loss of generality, $\eta$ can be chosen such that $H$ is admissible. From $\eta \in \mathcal{H}^{\tilde{K}}$, we have that $\Delta H_{t}=\eta_{t}-\eta_{t-1} \in-\tilde{K}_{t}=\left(-\tilde{K}_{t}^{*}\right)^{*}$. In particular, this implies $\Delta H_{t} \cdot \hat{S}_{t} \leq 0 \hat{\mathcal{P}}$-q.s. and therefore that

$$
\delta_{t}:=\Delta H_{t}^{d}+\sup _{\theta \in \Theta_{t}} \sum_{i=1}^{d-1} \Delta H_{t}^{i} \hat{S}_{t}^{i}(\cdot, \theta) \leq 0 \quad \mathcal{P} \text {-q.s. }
$$

Consider the new strategy $\tilde{\eta}$ with $\tilde{\eta}_{t}=\eta_{t}-e_{d} \sum_{u=0}^{t} \delta_{u}$ for $t \in \mathcal{I}$. Since $\Delta \tilde{H}_{t}^{i}=\Delta H_{t}^{i}$ for $i=1, \ldots, d-1$ and $-\Delta \tilde{H}_{t}^{d}=-\Delta H_{t}^{d}+\delta_{t}$, the self-financing condition follows from (4.4). Moreover, using again (4.4), we also have $\left(\tilde{\eta}_{t}-\tilde{\eta}_{t-1}\right) \cdot x \leq 0$ for any $x \in \tilde{K}_{t}^{*, 0}$, which implies that $\tilde{\eta}_{t}-\tilde{\eta}_{t-1} \in-\tilde{K}_{t}$. Finally, $\tilde{\eta}_{t}$ is in $\mathcal{L}^{0}\left(\overline{\mathcal{F}}_{t}^{u} ; C_{t}\right)$ since it coincides with $\eta_{t}$ in the first $d-1$ coordinates and the last one is unconstrained (see the comment after Theorem 2.4). We conclude by observing 
that $y e_{d}+\tilde{\eta}_{T-1}-G=y e_{d}+\eta_{T-1}-e_{d} \sum_{t=0}^{T-1} \delta_{t}-G$ is in $\tilde{K}_{T}$ since $\sum_{t=0}^{T-1} \delta_{t} \leq 0$ $\mathcal{P}$-q.s. and $\tilde{K}_{T}$ is a convex cone containing $\mathbb{R}_{+}^{d}$.

" $\leq$ ": If the set of superhedging strategies for $G \cdot \hat{S}_{T}$ is empty, then $\hat{\pi}\left(G \cdot \hat{S}_{T}\right)=\infty$ and the inequality holds trivially. Suppose that $(y, H) \in \mathbb{R} \times \hat{\mathcal{H}}$ is a superhedge for $G \cdot \hat{S}_{T}$. We set $k_{T}:=0, k_{t}:=H_{t}-H_{t+1}$ and $\eta_{t}:=\sum_{u=0}^{t}-k_{u}$ for any $t \in \mathcal{I}$. Suppose first $\mathbb{P}\left[k_{t}^{d}=\infty\right]>0$ for some $\mathbb{P} \in \mathcal{P}$ and $t \in \mathcal{I}$. From Lemma 4 .4, for any $n \in \mathbb{N}$, there exists $\hat{\mathbb{P}}^{n} \in \hat{\mathcal{P}}$ such that

$$
-\left(H_{t+1}^{d}-H_{t}^{d}\right)(\omega) \geq \sum_{i=1}^{d-1}-k_{t}^{i} \hat{S}_{t}^{i} \geq n \quad \hat{\mathbb{P}}^{n} \text {-a.s. }
$$

where the first inequality follows from (4.1). Note that the term $H_{t}^{d}-H_{t+1}^{d}$ is the cost of rebalancing at time $t$ the strategy $H$ which superhedges $G \cdot \hat{S}_{T}$ under $\hat{\mathbb{P}}^{n}$. Since $n$ is arbitrary and $\hat{\mathbb{P}}^{n} \in \hat{\mathcal{P}}$ for any $n \in \mathbb{N}$, we deduce that $\hat{\pi}\left(G \cdot \hat{S}_{T}\right)=\infty$ and the inequality holds trivially.

For the rest of the proof, we suppose that $k_{t}^{d}$ is pointwise finite for any $t \in \mathcal{I}$ (indeed, the $\mathcal{P}$-q.s. version $k_{t}^{d} \mathbf{1}_{\left\{k_{t}^{d}<\infty\right\}}$ is again universally measurable). From (4.4), we have $k_{t}(\omega) \cdot x \geq 0$ for any $x \in \tilde{K}_{t}^{*, 0}$, which implies $k_{t}(\omega) \in \tilde{K}_{t}(\omega)$. We now rewrite the superhedging property of $(y, H)$ in terms of $(y, \eta)$. For any $(\omega, \theta)$ outside a $\hat{\mathcal{P}}$-polar set, we have

$$
\begin{aligned}
0 & \leq y+\sum_{t=0}^{T-1} H_{t+1}(\omega) \cdot\left(\hat{S}_{t+1}-\hat{S}_{t}\right)(\omega, \theta)-G(\omega) \cdot \hat{S}_{T}(\omega, \theta) \\
& =y+\sum_{t=0}^{T-1}-k_{t}(\omega) \cdot\left(\hat{S}_{T}-\hat{S}_{t}\right)(\omega, \theta)-G(\omega) \cdot \hat{S}_{T}(\omega, \theta) \\
& =\left(y e_{d}+\eta_{T}(\omega)-G(\omega)\right) \cdot \hat{S}_{T}(\omega, \theta)+\sum_{t=0}^{T-1} k_{t}(\omega) \cdot \hat{S}_{t}(\omega, \theta) .
\end{aligned}
$$

We claim that this implies

$$
0 \leq\left(y e_{d}+\eta_{T}(\omega)-G(\omega)\right) \cdot \hat{S}_{T}(\omega, \theta) \quad \hat{\mathcal{P}} \text {-q.s. }
$$

To prove the claim, observe that the first term in (4.5) depends on $\theta$ only through the last component $\theta_{T}$, whereas the second term in (4.5) depends only on the first $T-1$ components of $\theta$. Fix $n \in \mathbb{N}, \mathbb{P} \in \mathcal{P}$. From Lemma 4.4 and (4.4), there exists $\hat{\mathbb{P}}^{n} \in \hat{\mathcal{P}}$ such that $\left.\hat{\mathbb{P}}^{n}\right|_{\Omega}=\mathbb{P}$ and

$$
k_{t} \cdot \hat{S}_{t}=\sum_{i=1}^{d-1} k_{t}^{i} \hat{S}_{t}^{i}+k_{t}^{d} \leq \frac{1}{n} \quad \hat{\mathbb{P}}^{n} \text {-a.s. }
$$

Since $n \in \mathbb{N}$ and $\mathbb{P} \in \mathcal{P}$ are arbitrary, we deduce that (4.6) holds and the claim is proved. It remains to show that for $\xi=y e_{d}+\eta_{T}-G$, we have

$$
\xi \cdot \hat{S}_{T} \geq 0 \hat{\mathcal{P}} \text {-q.s. } \quad \Longrightarrow \quad \xi \in \tilde{K}_{T} \mathcal{P} \text {-q.s. }
$$


Suppose by contradiction that there exist a set $A$ and a probability $\mathbb{P} \in \mathcal{P}$ such that $\mathbb{P}[A]>0$ and $\xi(\omega) \notin \tilde{K}_{T}(\omega)$ for any $\omega \in A$. Without loss of generality, we may take a Borel-measurable version of $\xi$. Recall that $\tilde{K}_{T}=K_{T}$ is assumed to be Borelmeasurable, so that

$$
B:=\left\{(\omega, y) \in \Omega \times \mathbb{R}^{d}: \xi(\omega) \cdot y<0\right\} \cap \operatorname{graph}\left(\operatorname{int}\left(\tilde{K}_{T}^{*}\right)\right)
$$

is Borel-measurable by [13, Lemma A.1]. Moreover, its projection on $\Omega$ contains $A$. As $B$ is Borel, from the Jankov-von Neumann theorem, there exists a universally measurable $s_{T}: \Omega \rightarrow \mathbb{R}^{d}$ with $\operatorname{graph}\left(s_{T}\right) \subseteq B$. Since $\operatorname{graph}\left(s_{T}\right) \subseteq \operatorname{graph}\left(\operatorname{int}\left(K_{T}^{*}\right)\right)$, we can normalise with respect to the last component, and from [24, Theorem 14.16], there exists an $\mathcal{F}_{T}^{u}$-measurable random vector $\bar{\theta}_{T}$ satisfying

$$
\xi(\omega) \cdot \hat{S}_{T}\left(\omega, \bar{\theta}_{T}(\omega)\right)<0, \quad \forall \omega \in A .
$$

Take also $\bar{\theta}_{t}$ to be a selector of $\Theta_{t}$ for any $0 \leq t \leq T-1$. For any of the above selectors, we take a Borel-measurable version. Consider now the probability measure $\hat{\mathbb{P}} \in \hat{\mathcal{P}}$ obtained from the kernels

$$
\hat{P}_{t}\left(\omega_{0}, \ldots, \omega_{t} ; \omega^{\prime}, \theta^{\prime}\right):=P_{t}\left[\omega_{0}, \ldots, \omega_{t} ; d \omega^{\prime}\right] \otimes \delta_{\bar{\theta}_{t+1}\left(\omega_{0}, \ldots, \omega_{t} ; \omega^{\prime}\right)}\left(\theta^{\prime}\right),
$$

with $\hat{P}_{-1}:=P_{-1} \otimes \delta_{\bar{\theta}_{0}}$ for an arbitrary $P_{-1} \in \mathfrak{P}\left(\Omega_{0}\right)$. Note that $\hat{\mathbb{P}} \in \hat{\mathcal{P}}$ and $\left.\hat{\mathbb{P}}\right|_{\Omega}=\mathbb{P}$. By construction,

$$
\hat{\mathbb{P}}\left[\xi \cdot \hat{S}_{T}<0\right] \geq \mathbb{P}[A]>0,
$$

which contradicts the hypothesis.

\subsection{Equality of the dual problems}

From [8, Lemma 5.7], any $\hat{\mathbb{Q}} \in \hat{\mathcal{Q}}$ admits a disintegration $\left(\hat{Q}_{t}\right)_{t=0, \ldots, T-1}$, where $\hat{Q}_{t}$ is a universally measurable selector of

$$
\begin{aligned}
\hat{\mathcal{Q}}_{t}(\hat{\omega}):=\left\{\hat{\mathbb{P}} \in \mathfrak{P}\left(\hat{\Omega}_{0}\right):\right. & \hat{\mathbb{P}} \ll \hat{\mathcal{P}}_{t}(\omega), \quad \mathbb{E}_{\hat{\mathbb{P}}}\left[\left|\Delta \hat{S}_{t}(\hat{\omega} ; \cdot)\right|\right]<\infty \text { and } \\
& \left.\mathbb{E}_{\hat{\mathbb{P}}}\left[y \cdot \Delta \hat{S}_{t}(\hat{\omega} ; \cdot)\right] \leq 0, \forall y \in C_{t}(\omega)\right\}, \quad \hat{\omega} \in \hat{\Omega}_{t},
\end{aligned}
$$

for $t=0, \ldots, T-1$. Analogously, the set of normalised SCPSs $\tilde{\mathcal{S}}^{0}$ for the market $\tilde{\mathbb{K}}$ is composed of couples $(Z, \mathbb{Q})$ for which the disintegration $\left(Q_{t}\right)_{t=0, \ldots, T-1}$ of $\mathbb{Q}$ satisfies

$$
\mathbb{E}_{Q_{t}}\left[\left|\Delta Z_{t}(\omega ; \cdot)\right|\right]<\infty \quad \text { and } \quad \mathbb{E}_{Q_{t}}\left[y \cdot \Delta Z_{t}\right] \leq 0, \forall y \in C_{t}(\omega),
$$

for $t=0, \ldots, T-1$.

Proposition 4.6 For any random vector $G \in \mathcal{F}^{u}$,

$$
\sup _{(Z, \mathbb{Q}) \in \tilde{\mathcal{S}}^{0}} \mathbb{E}_{\mathbb{Q}}\left[G \cdot Z_{T}\right]=\sup _{\hat{\mathbb{Q}} \in \hat{\mathcal{Q}}} \mathbb{E}_{\hat{\mathbb{Q}}}\left[G \cdot \hat{S}_{T}\right] .
$$


Proof Suppose that $(Z, \mathbb{Q}) \in \tilde{\mathcal{S}}^{0}$. By construction, $\hat{S}_{t}$ is a Carathéodory map. From the implicit mapping theorem (see [24, Theorem 14.16]), there exists an $\mathbb{F}$-adapted process $\left(\theta_{t}\right)_{t \in \mathcal{I}}$ with $\theta_{t}: \Omega_{t} \rightarrow \mathbb{R}^{d-1}$ and such that

$$
\hat{S}_{t}\left(\omega, \theta_{t}(\omega)\right)=Z_{t}(\omega) .
$$

Denote by $\left(Q_{t}\right)_{t \in \mathcal{I}_{-1}}$ the collection of conditional probabilities of $\mathbb{Q}$ given $\mathcal{F}_{t-1}$, extended to $t=-1$ with an arbitrary $Q_{-1} \in \mathfrak{P}\left(\Omega_{0}\right)$. We use $\delta_{\theta_{t}}$ as a stochastic kernel and construct the probability $\hat{\mathbb{Q}}:=\left(Q_{-1} \otimes \delta_{\theta_{0}}\right) \otimes \cdots \otimes\left(Q_{T-1} \otimes \delta_{\theta_{T}}\right)$. Since $(Z, \mathbb{Q})$ satisfies $(4.8)$ and $(4.9)$ holds, we deduce that $\hat{\mathbb{Q}} \in \hat{\mathcal{Q}}$. Moreover, it is clear that $\mathbb{E}_{\mathbb{Q}}\left[G \cdot Z_{T}\right]=\mathbb{E}_{\hat{\mathbb{Q}}}\left[G \cdot \hat{S}_{T}\right]$.

Conversely, suppose $\hat{\mathbb{Q}} \in \hat{\mathcal{Q}}$. We define $(Z, \mathbb{Q})$ via $\mathbb{Q}:=\hat{\mathbb{Q}}_{\Omega}$ and $Z_{t}:=\mathbb{E}_{\hat{\mathbb{Q}}}\left[\hat{S}_{t} \mid \mathcal{F}_{t}\right]$ for every $t \in \mathcal{I}$. Denote by $\left(\hat{Q}_{t}\right)_{t \in \mathcal{I}_{-1}}$ (respectively $\left.\left(Q_{t}\right)_{t \in \mathcal{I}_{-1}}\right)$ the disintegration of $\hat{\mathbb{Q}}$ (respectively of $\mathbb{Q}$ ). From $\mathbb{E}_{\hat{Q}_{t}}\left[y \cdot \Delta \hat{S}_{t+1}(\hat{\omega} ; \cdot)\right] \leq 0$ for any $y \in C_{t}$ and from the fact that $C_{t}$ is $\mathcal{F}_{t}$-measurable, we deduce that $Q_{t}$ satisfies (4.8) for any $t=0, \ldots, T-1$. Moreover, since $\hat{\mathbb{Q}} \ll \hat{\mathcal{P}}$, we obtain by the definition of $\hat{\mathcal{P}}$ that $\mathbb{Q} \ll \mathcal{P}$ and $Z_{t}$ takes values in $\operatorname{int}\left(\tilde{K}_{t}^{*}\right) \mathbb{Q}$-a.s. for any $t \in \mathcal{I}$. We conclude that $(Z, \mathbb{Q}) \in \tilde{\mathcal{S}}^{0}$. Moreover, we obviously have $\mathbb{E}_{\mathbb{Q}}\left[G \cdot Z_{T}\right]=\mathbb{E}_{\hat{\mathbb{Q}}}\left[G \cdot \hat{S}_{T}\right]$.

\subsection{Proof of Theorem 2.4}

We are now ready to prove the main result. Note that from Lemmas 4.1 and 4.5, we can only deduce the equality of the primal problems if one restricts to consistent trading in the enlarged market (compare with (4.3)). It remains to show that the same price is obtained with randomised strategies as defined in (4.2); in other words, we need to prove that $\hat{\pi}\left(G \cdot \hat{S}_{T}\right)=\hat{\pi}^{r}\left(G \cdot \hat{S}_{T}\right)$. Denote by $U S A\left(\hat{\Omega}_{t}, t\right)$ the class of upper semianalytic functions $g: \hat{\Omega}_{t} \rightarrow \mathbb{R}$ which depend on $\theta$ only through $\theta_{t}$, i.e.,

$$
g(\omega, \theta)=g\left(\omega^{\prime}, \theta^{\prime}\right), \quad \forall(\omega, \theta),\left(\omega^{\prime}, \theta^{\prime}\right) \in \hat{\Omega}_{t} \text { with } \omega=\omega^{\prime} \text { and } \theta_{t}=\theta_{t}^{\prime} .
$$

We first obtain results for $T=1$ which constitute the building blocks for the general case.

Proposition 4.7 Suppose $T=1$ and $g \in U S A\left(\hat{\Omega}_{T}, T\right)$. If $\mathrm{NA}(\hat{\mathcal{P}})$ holds true, then $\hat{\pi}(g)=\hat{\pi}^{r}(g)$.

Proof The inequality $\hat{\pi}(g) \geq \hat{\pi}^{r}(g)$ is trivial. For the converse, let $B_{n}(0)$ be the closed ball in $\mathbb{R}^{d}$ with centre in 0 and radius $n \in \mathbb{N}$. The intersection $\tilde{K}_{0}^{*, 0} \cap B_{n}(0)$ is a compact set of the form $O^{n} \times\{1\}$ for a compact subset $O^{n}$ of $\mathbb{R}^{d-1}$. Recall the definition of $\hat{\mathcal{P}}_{0}$ from (3.5) and let $P_{-1} \in \mathfrak{P}\left(\Omega_{0}\right)$ be arbitrary. ${ }^{2}$ We define

$$
\hat{\mathcal{P}}^{n}:=\left\{\left(P_{-1} \otimes \delta_{\theta}\right) \otimes \hat{\mathbb{P}}: \theta \in O^{n}, \hat{\mathbb{P}} \in \hat{\mathcal{P}}_{0}\right\} \subseteq \hat{\mathcal{P}} .
$$

Denote by $\hat{\pi}_{n}$ and $\hat{\pi}_{n}^{r}$ the analogues of $\hat{\pi}$ and $\hat{\pi}^{r}$ in (4.2) and (4.3) with $\hat{\mathcal{P}}^{n}$ replacing $\hat{\mathcal{P}}$ and note that by construction, $\left(\hat{\pi}_{n}(g)\right)_{n \in \mathbb{N}}$ and $\left(\hat{\pi}_{n}^{r}(g)\right)_{n \in \mathbb{N}}$ are increasing

\footnotetext{
${ }^{2}$ Recall that the cone $\tilde{K}_{0}^{*, 0}$ is constant in $\omega$. Thus in the enlarged market, the only relevant variable is $\theta$.
} 
sequences bounded from above by $\hat{\pi}(g)$ and $\hat{\pi}^{r}(g)$, respectively. We now use a minimax argument as in Bouchard et al. [11] to deduce that

$$
\begin{aligned}
\hat{\pi}_{n}(g) & =\inf _{H \in C_{0}} \sup _{\theta \in O^{n}} \sup _{\hat{\mathbb{P}} \ll \hat{\mathcal{P}}_{0}} \mathbb{E}_{\hat{\mathbb{P}}}\left[g-H \cdot\left(\hat{S}_{1}-\hat{S_{0}}\right)\right] \\
& =\sup _{\theta \in O^{n}} \inf _{H \in C_{0}} \sup _{\hat{\mathbb{P}} \ll \hat{\mathcal{P}}_{0}} \mathbb{E}_{\hat{\mathbb{P}}}\left[g-H \cdot\left(\hat{S}_{1}-\hat{S_{0}}\right)\right] \\
& =\hat{\pi}_{n}^{r}(g) .
\end{aligned}
$$

To justify the above, it is sufficient to observe that the function

$$
(H, \theta) \mapsto \sup _{\hat{\mathbb{P}} \ll \hat{\mathcal{P}}_{0}} \mathbb{E}_{\hat{\mathbb{P}}}\left[g-H \cdot\left(\hat{S}_{1}-\hat{S_{0}}\right)\right]=\sup _{\hat{\mathbb{P}} \ll \hat{\mathcal{P}}_{0}} \mathbb{E}_{\hat{\mathbb{P}}}\left[g-H \cdot \hat{S}_{1}\right]-H \cdot \hat{S}_{0}(\omega, \theta)
$$

is convex in $H$ for fixed $\theta$ and affine in $\theta$ for fixed $H$. We can thus apply the minimax theorem of [25, Corollary 2]. If $\hat{\pi}^{r}(g) \geq \lim _{n \rightarrow \infty} \hat{\pi}_{n}^{r}(g)=\infty$, then $\hat{\pi}^{r}(g) \geq \hat{\pi}(g)$ holds trivially and the proof is complete. Suppose the limit is finite. Let $\varepsilon>0$ be arbitrary and for any $n \in \mathbb{N}$, let $H_{n} \in C_{0}$ be an $\varepsilon$-optimal strategy for $\hat{\pi}_{n}(g)$, i.e.,

$$
\hat{\pi}_{n}(g)+\varepsilon+H_{n} \cdot\left(\hat{S}_{1}\left(\omega, \theta_{1}\right)-\hat{S_{0}}\left(\omega, \theta_{0}\right)\right) \geq g\left(\omega, \theta_{1}\right)
$$

for any $\left(\omega, \theta_{1}\right)$ outside a $\hat{\mathcal{P}}_{0}$-polar set and for any $\theta_{0} \in O^{n}$. If $\left(H_{n}\right)_{n \in \mathbb{N}} \subseteq C_{0}$ is bounded, it admits a convergent subsequence. Denote by $\bar{H}$ its limit. From (4.10), $O^{n} \times\{1\} \uparrow \tilde{K}_{0}^{*, 0}$ and because $\hat{\pi}^{r}(g) \geq \lim _{n \rightarrow \infty} \hat{\pi}_{n}^{r}(g)=\lim _{n \rightarrow \infty} \hat{\pi}_{n}(g)$, we get

$$
\hat{\pi}^{r}(g)+\varepsilon+\bar{H} \cdot\left(\hat{S}_{1}-\hat{S}_{0}\right) \geq g \quad \hat{\mathcal{P}} \text {-q.s. }
$$

from which $\hat{\pi}(g) \leq \hat{\pi}^{r}(g)+\varepsilon$. We show now that if $\left(H_{n}\right)_{n \in \mathbb{N}} \subseteq C_{0}$ is unbounded, it contradicts $\mathrm{NA}(\hat{\mathcal{P}})$. This together with $\varepsilon>0$ being arbitrary yields the desired inequality. To obtain the contradiction, divide by $c_{n}:=\left\|H_{n}\right\|$ both sides of (4.10). Since $\hat{\pi}_{n}(g)=\hat{\pi}_{n}^{r}(g)$ is assumed to be bounded, $\left(\hat{\pi}_{n}(g) / c_{n}\right)$ converges to 0 . As $\left(H_{n} / c_{n}\right)$ belongs to the compact unit sphere of $\mathbb{R}^{d}$, there exists $\bar{H}$ with $\|\bar{H}\|=1$ such that $H_{n} / c_{n} \rightarrow \bar{H}$ (up to extracting a subsequence). Note that $\bar{H} \in C_{0}$ since $C_{0}$ is a closed cone. By the same argument as above, this implies that

$$
\bar{H} \cdot\left(\hat{S}_{1}-\hat{S_{0}}\right) \geq 0 \quad \hat{\mathcal{P}} \text {-q.s. }
$$

Recalling (3.7), the condition $\mathrm{NA}(\hat{\mathcal{P}})$ implies $\bar{H}=0$, which is a contradiction since $\|\bar{H}\|=1$. This concludes the proof.

Note that if $G: \Omega \rightarrow \mathbb{R}^{d}$ is a Borel-measurable vector, $G \cdot \hat{S}_{T}: \hat{\Omega} \rightarrow \mathbb{R}$ is a Borelmeasurable function which depends on $\theta$ only through $\theta_{T}$. In particular, Proposition 4.7 together with Lemmas 4.1 and 4.5 yields $\pi_{K}(G)=\hat{\pi}^{r}\left(G \cdot \hat{S}_{T}\right)$.

Proof of Theorem 2.4 for $T=1$ By Proposition 3.9, $\mathrm{NA}^{\mathrm{s}}(\mathcal{P})$ implies $\mathrm{NA}(\hat{\mathcal{P}})$ for the enlarged market. From Lemmas 4.1 and 4.5 and Proposition 4.7, we get 
$\pi_{\mathbb{K}}(G)=\hat{\pi}^{r}\left(G \cdot \hat{S}_{T}\right)$ which is the superhedging price of $G \cdot \hat{S}_{T}$ in the enlarged market. We show that

$$
\pi_{\mathbb{K}}(G)=\hat{\pi}^{r}\left(G \cdot \hat{S}_{T}\right)=\sup _{\hat{\mathbb{Q}} \in \hat{\mathcal{Q}}} \mathbb{E}_{\hat{\mathbb{Q}}}\left[G \cdot \hat{S}_{T}\right]=\sup _{(Z, \mathbb{Q}) \in \tilde{\mathcal{S}}^{0}} \mathbb{E}_{\mathbb{Q}}\left[G \cdot Z_{T}\right] \leq \sup _{(Z, \mathbb{Q}) \in \mathcal{S}^{0}} \mathbb{E}_{\mathbb{Q}}\left[G \cdot Z_{T}\right] .
$$

Indeed, the second equality follows from [8, Theorem 4.3] after observing that when $C_{0}$ is a cone, $A^{\mathbb{Q}}$ in that paper is finite if and only if $\hat{\mathbb{Q}} \in \hat{\mathcal{Q}}$. The third equality follows from Proposition 4.6 and the last inequality follows from $\tilde{\mathcal{S}}^{0} \subseteq \mathcal{S}^{0}$. The converse inequality follows from standard arguments. From [8, Theorem 4.3], a cheapest superhedging strategy exists in the enlarged market when the price is finite. The proofs of Lemmas 4.1 and 4.5 provide the construction of a cheapest strategy in the original market.

We now analyse the multi-period case. From [8, Lemma 3.4], $\hat{\mathcal{Q}}_{t}$ as in (4.7) has analytic graph for every $0 \leq t \leq T-1$. Given $g_{t+1} \in U S A\left(\hat{\Omega}_{t+1}, t+1\right)$, we define

$$
\begin{aligned}
g_{t}: \hat{\Omega}_{t} \rightarrow \overline{\mathbb{R}}, \quad g_{t}(\hat{\omega})=\sup _{\hat{\mathbb{Q}} \in \hat{\mathcal{Q}}_{t}(\hat{\omega})} \mathbb{E}_{\hat{\mathbb{Q}}}\left[g_{t+1}\right], \\
g_{t}^{\prime}: \Omega_{t} \times \mathbb{R}^{d} \rightarrow \overline{\mathbb{R}}, \quad g_{t}^{\prime}(\omega, h)=\sup _{\theta \in \Theta_{t}(\omega)}\left(g_{t}(\omega, \theta)-h \cdot \hat{S}_{t}(\omega, \theta)\right),
\end{aligned}
$$

with $\Theta_{t}$ as in (3.3). Then $g_{t}$ is in $U S A\left(\hat{\Omega}_{t}, t\right)$. Indeed, the measurability property follows exactly from the same argument as in the first lines of the proof of [12, Lemma 4.10]. Moreover, $g_{t}$ depends on $\theta$ only through $\hat{S}_{t}$, thus only through $\theta_{t}$ (see (3.2)). Recall now that the sum of two upper semianalytic functions is again upper semianalytic (see e.g. [10, Lemma 7.30]). Since $\hat{S}_{t}$ is Borel-measurable, we deduce that $g_{t}-h \cdot \hat{S}_{t}$ is an upper semianalytic function of $(\omega, h, \theta)$. From Lemma 3.1 and [10, Proposition 7.47], we deduce that $g_{t}^{\prime}$ is upper semianalytic. Let $\tilde{\mathcal{P}}_{t}$ be the set of probabilities on $\Omega_{t} \times \mathbb{R}^{d-1} \times \hat{\Omega}_{0}$ given by

$$
\tilde{\mathcal{P}}_{t}(\omega):=\left\{\left(\delta_{\omega} \otimes \delta_{\theta}\right) \otimes \hat{\mathbb{P}}:(\omega, \theta) \in \operatorname{graph}\left(\Theta_{t}\right), \hat{\mathbb{P}} \in \hat{\mathcal{P}}_{t}(\omega)\right\}, \quad \omega \in \Omega_{t} .
$$

Recall that the multifunctions $\hat{\mathcal{P}}_{t}$ and $\delta_{\Theta}$ from Corollary 3.2 have analytic graphs. Since the map $x \mapsto \delta_{x}$ is an embedding and the map $(P, Q) \mapsto P \otimes Q$ is continuous (see [10, Lemma 7.12]), it follows that also $\tilde{\mathcal{P}}_{t}$ has analytic graph.

Lemma 4.8 For any $0 \leq t \leq T-1$, the function $f: \Omega_{t} \times \mathbb{R}^{d} \times \mathbb{R}^{d} \rightarrow \overline{\mathbb{R}}$ defined as

$$
f(\omega, h, x)=\sup _{\tilde{\mathbb{P}} \ll \tilde{\mathcal{P}}_{t}(\omega)} \mathbb{E}_{\tilde{\mathbb{P}}}\left[g_{t+1}-h \cdot \hat{S}_{t}-x \cdot\left(\hat{S}_{t+1}-\hat{S}_{t}\right)\right]
$$

is a universally measurable normal integrand.

Proof Denote by $f^{\tilde{\mathbb{P}}}(\omega, h, x)$ the functions on the right-hand side of (4.13) over which the supremum is taken. From [24, Corollary 14.41], we need to check that

(1) for any $(\omega, h) \in \Omega_{t} \times \mathbb{R}^{d}$, the function $f(\omega, h, \cdot)$ is lower semicontinuous; 
(2) for any $x \in \mathbb{R}^{d}$, there exists $\varepsilon^{\prime}>0$ such that for all $\varepsilon \in\left(0, \varepsilon^{\prime}\right)$, the function

$$
\Psi_{\varepsilon}:(\omega, h) \mapsto \inf _{\tilde{x} \in B_{\varepsilon}(x)} f(\omega, h, \tilde{x})
$$

is universally measurable, where $B_{\varepsilon}(x)$ denotes the closed ball of radius $\varepsilon$ centred in $x$.

Since $f^{\tilde{\mathbb{P}}}(\omega, h, \cdot)$ is continuous for every $\tilde{\mathbb{P}}$ and the pointwise supremum of continuous functions is lower semicontinuous, the first claim follows. Consider an arbitrary $\varepsilon>0$. We first show that for any $(\omega, h)$, we have

$$
\Psi_{\varepsilon}(\omega, h)=\sup _{\tilde{\mathbb{P}} \ll \tilde{\mathcal{P}}_{t}(\omega)} \inf _{\tilde{x} \in B_{\varepsilon}(x)} f^{\tilde{\mathbb{P}}}(\omega, h, \tilde{x}) .
$$

This follows from the application of a minimax theorem (see e.g. [25, Corollary 2]). Indeed, $B_{\varepsilon}(x)$ is a compact set and for fixed $\tilde{x}$, the map $f^{\tilde{\mathbb{P}}}(\omega, h, \tilde{x})$ is linear (hence concave) in $\tilde{\mathbb{P}}$. On the other hand, $\left\{\tilde{\mathbb{P}} \ll \tilde{\mathcal{P}}_{t}(\omega)\right\}$ is a convex set and for fixed $\tilde{\mathbb{P}}$, the map $f^{\tilde{\mathbb{P}}}(\omega, h, \tilde{x})$ is affine (hence convex) and continuous in $\tilde{x}$. We can rewrite $\inf _{\tilde{x} \in B_{\varepsilon}(x)} f^{\tilde{\mathbb{P}}}(\omega, h, \tilde{x})=f_{1}(\omega, h, \tilde{\mathbb{P}})+f_{2}(\omega, \tilde{\mathbb{P}})$, where

$$
f_{1}=\mathbb{E}_{\tilde{\mathbb{P}}}\left[g_{t+1}-h \cdot \hat{S}_{t}\right], \quad f_{2}=-\sup _{\tilde{x} \in B_{\varepsilon}(x)} \mathbb{E}_{\tilde{\mathbb{P}}}\left[\tilde{x} \cdot\left(\hat{S}_{t+1}-\hat{S}_{t}\right)\right]
$$

$f_{1}$ is an upper semianalytic function on $\Omega \times \mathbb{R}^{d} \times \mathfrak{P}(\Omega)$ (see [12, Lemma 4.10]). We claim that $f_{2}$ is a Borel-measurable function (hence upper semianalytic). Given the claim, we observe that $f_{1}+f_{2}$ is again upper semianalytic. Moreover, by the same argument as for the measurability of (4.12) above, we can conclude that the function $\Psi_{\varepsilon}=\sup _{\tilde{\mathbb{P}} \ll \tilde{\mathcal{P}}_{t}(\omega)}\left(f_{1}+f_{2}\right)$ is upper semianalytic on $\Omega \times \mathbb{R}^{d}$ and therefore universally measurable. This proves the second property.

To conclude the proof, it is enough to show that $f_{2}$ is Borel-measurable. To see this, observe that the function $\mathbb{E}_{\mathbb{P}}\left[\tilde{x} \cdot\left(\hat{S}_{t+1}(\omega ; \cdot)-\hat{S}_{t}(\omega ; \cdot)\right)\right]$ is measurable in $(\omega, \mathbb{P})$ and continuous in $\tilde{x}$, i.e., it is a Carathéodory map. From [24, Example 14.15], its composition with $B_{\varepsilon}(x)$ yields a Borel-measurable multifunction $A$ such that $\sup A=-f_{2}$. To conclude, observe that for an arbitrary $c \in \mathbb{R}$,

$$
\left\{(\omega, \mathbb{P}): f_{2}<c\right\}=\{(\omega, \mathbb{P}): A \cap(-c, \infty) \neq \emptyset\}
$$

is a Borel set from the measurability of $A$.

Remark 4.9 Note that for any $\omega \in \Omega_{t}$, the right-hand side of (4.13) is equal to $\inf \left\{K \in \mathbb{R}: X \leq K \hat{\mathcal{P}}_{t}(\omega)\right.$-q.s. $\}$, where $X$ is the random variable inside the expectation. In particular, this is equal to the minimal amount at time $t$ for which the strategy $x$ is a superhedge for $g_{t+1}$, given that $h$ is the strategy used at time $t-1$. Moreover, by the construction of $\tilde{\mathcal{P}}_{t}$, the strategy $x$ with the initial amount $f(\omega, h, x)$ is a (conditional) superhedging strategy which depends only on the event $\omega$ and not on the event $(\omega, \theta)$. In the terminology of Definition 4.2 , this construction provides consistent strategies. 
Recall that $\mathrm{NA}\left(\hat{\mathcal{P}}_{t}(\omega)\right)$ is the conditional version of $\mathrm{NA}(\hat{\mathcal{P}})$ (see Theorem 3.8).

Proposition 4.10 Let $0 \leq t \leq T-1$ and assume $\mathrm{NA}\left(\hat{\mathcal{P}}_{t}(\omega)\right)$. There exist a universally measurable map $\varphi: \Omega_{t} \times \mathbb{R}^{d} \rightarrow \mathbb{R}^{d}$ and a $\mathcal{P}$-polar set $N$ such that for any $(\omega, h) \in N^{c} \times \mathbb{R}^{d}$, we have

$$
g_{t}^{\prime}(\omega, h)+h \cdot \hat{S}_{t}(\omega)+\varphi(\omega, h) \cdot\left(\hat{S}_{t+1}(\omega ; \cdot)-\hat{S}_{t}(\omega)\right) \geq g_{t+1}(\omega ; \cdot) \quad \tilde{\mathcal{P}}_{t}(\omega)-q . s .
$$

and $g_{t}^{\prime}(\omega, h)>-\infty$.

Proof Define the consistent conditional superhedging price of $g_{t+1}$ given $(\omega, h)$ as the map

$$
\begin{aligned}
& (\omega, h) \mapsto \inf \left\{y \in \mathbb{R}: \exists H \in C_{t}(\omega)\right. \text { with } \\
& \left.\qquad y+h \cdot \hat{S}_{t}(\omega)+H \cdot\left(\hat{S}_{t+1}(\omega ; \cdot)-\hat{S}_{t}(\omega)\right) \geq g_{t+1}(\omega ; \cdot) \tilde{\mathcal{P}}_{t}(\omega) \text {-q.s. }\right\} .
\end{aligned}
$$

The fact that the function $g_{t}^{\prime}(\omega, h)$ defined in (4.12) coincides with the consistent conditional superhedging price of $g_{t+1}$ follows from the same minimax argument as for Proposition 4.7 and [8, Theorem 4.3]. Moreover, from Theorem 3.8, NA $\left(\hat{\mathcal{P}}_{t}(\omega)\right)$ holds outside a polar set $N$. Again from [8, Theorem 4.3], we have $g_{t}^{\prime}(\omega, h)>-\infty$ on $N^{c} \times \mathbb{R}^{d}$ and the infimum is attained. It remains to show that a superhedging strategy can be chosen in a measurable way. From Lemma 4.8, the map $f$ defined in (4.13) is a universally measurable normal integrand. From [24, Proposition 14.33] and recalling that $g_{t}^{\prime}$ is upper semianalytic (hence universally measurable), the map

$$
\Psi(\omega, h):=\left\{x \in \mathbb{R}^{d}: f(\omega, h, x) \leq g_{t}^{\prime}(\omega, h)\right\}
$$

is a closed-valued universally measurable multifunction. The desired $\varphi$ is any measurable selector of $\Psi$ (which exists from e.g. [24, Corollary 14.6]).

Proof of Theorem 2.4 for $T>1$ We first show that for any $g \in U S A\left(\hat{\Omega}_{T}, T\right)$,

$$
\hat{\pi}(g)=\hat{\pi}^{r}(g)=\sup _{\hat{\mathbb{Q}} \in \hat{\mathcal{Q}}} \mathbb{E}_{\hat{\mathbb{Q}}}[g] .
$$

For $T=1$, (4.14) follows from Proposition 4.7 and [8, Theorem 4.3]. We prove the general case by induction. Suppose that (4.14) has been proved for $T=1, \ldots, t$. Denote by $\hat{\pi}_{u}$ the (consistent) superhedging price if the terminal time is $u$. In particular, $\hat{\pi}_{T}=\hat{\pi}$ defined in (4.3). Let $g_{t+1} \in U S A\left(\hat{\Omega}_{t+1}, t+1\right)$ and define $g_{t}$ and $g_{t}^{\prime}$ as in (4.11) and (4.12), respectively. We claim that $\hat{\pi}_{t+1}\left(g_{t+1}\right) \leq \hat{\pi}_{t}\left(g_{t}\right)$. Denote by $\left.\hat{\mathcal{P}}\right|_{t}$ the restriction of $\hat{\mathcal{P}}$ to $\hat{\Omega}_{t}$, and similarly for $\left.\hat{\mathcal{Q}}\right|_{t}$. Consider an arbitrary $(y, H) \in \mathbb{R} \times \hat{\mathcal{H}}^{r}$ satisfying $y+(H \cdot \hat{S})_{t} \geq\left. g_{t} \hat{\mathcal{P}}\right|_{t}$-q.s. By rewriting this inequality, we observe that

$$
y+(H \cdot \hat{S})_{t-1}-H_{t} \cdot \hat{S}_{t-1} \geq g_{t}-\left.H_{t} \cdot \hat{S}_{t} \quad \hat{\mathcal{P}}\right|_{t} \text {-q.s. },
$$

which in turn implies that

$$
y+(H \cdot \hat{S})_{t-1}-H_{t} \cdot \hat{S}_{t-1} \geq\left. g_{t}^{\prime}\left(\cdot, H_{t}\right) \quad \hat{\mathcal{P}}\right|_{t} \text {-q.s. }
$$


Given the strategy $\left(y, H_{1}, \ldots, H_{t}\right)$, Proposition 4.10 provides a universally measurable random vector $H_{t+1}=\varphi\left(\cdot, H_{t}\right)$ such that the strategy $\left(y, H_{1}, \ldots, H_{t}, H_{t+1}\right)$ satisfies $y+(H \cdot \hat{S})_{t+1} \geq g_{t+1} \hat{\mathcal{P}}$-q.s. Because $(y, H)$ above are arbitrary, the claim is proved. We deduce that

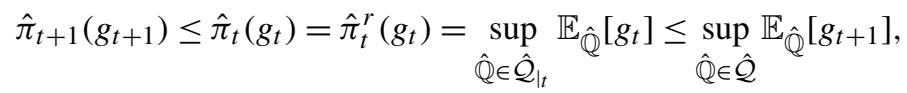

where the equalities follow from the induction hypothesis and the second inequality follows from a standard pasting argument. By definition, $\hat{\pi}_{t+1}^{r}\left(g_{t+1}\right) \leq \hat{\pi}_{t+1}\left(g_{t+1}\right)$; moreover, the inequality $\sup _{\hat{\mathbb{Q}} \in \hat{\mathcal{Q}}} \mathbb{E}_{\hat{\mathbb{Q}}}\left[g_{t+1}\right] \leq \hat{\pi}_{t+1}^{r}\left(g_{t+1}\right)$ is standard. We conclude that (4.14) holds for $T=t+1$. We now choose $g=G \cdot \hat{S}_{T}$, which is Borel-measurable by assumption. From Lemmas 4.1 and 4.5, Proposition 4.6 and (4.14), we deduce

$$
\pi_{\mathbb{K}}(G)=\sup _{(Z, \mathbb{Q}) \in \tilde{\mathcal{S}}^{0}} \mathbb{E}_{\mathbb{Q}}\left[G \cdot Z_{T}\right] \leq \sup _{(Z, \mathbb{Q}) \in \mathcal{S}^{0}} \mathbb{E}_{\mathbb{Q}}\left[G \cdot Z_{T}\right]
$$

Again, the converse inequality follows by standard arguments, and so the duality follows (recall also the discussion after Theorem 2.4). Finally, the attainment property in the frictionless market follows from [8, Theorem 6.1]. The proofs of Lemmas 4.1 and 4.5 provide the construction of a cheapest strategy in the original market.

\subsection{The case with options}

We now consider the case where a finite number of options $\varphi_{1}, \ldots, \varphi_{e}$ are available for semi-static trading. In this section, we show that this case can be embedded in the previous one. For any $k=1, \ldots, e$, we assume that $\varphi_{k}: \Omega \rightarrow \mathbb{R}^{d}$ is a Borelmeasurable function representing the terminal payoff of an option, in terms of physical units of an underlying $d$-dimensional asset. Any $\varphi_{k}$ has bid and ask prices at time 0 denoted, respectively, by $b_{k}$ and $a_{k}$. We set $\Phi:=\left(\varphi_{1}, \ldots, \varphi_{e},-\varphi_{1}, \ldots,-\varphi_{e}\right)$ with corresponding prices $p:=\left(a_{1}, \ldots, a_{e},-b_{1}, \ldots,-b_{e}\right)^{T} . \Phi$ takes values in $\mathbb{R}^{d \times m}$ and $p \in \mathbb{R}^{m}$ with $m:=2 e$. For ease of notation, we relabel the options and incorporate their price in the payoff so that $\Phi=\left(\phi_{1}-p_{1} e_{d}, \ldots, \phi_{m}-p_{m} e_{d}\right)$. In addition, we suppose that we are given a dynamic trading market $(\mathbb{K}, C)$ satisfying all the hypotheses of Sect. 2. An admissible strategy has the form $\bar{\eta}:=(\eta, \alpha)$, where $\eta \in \mathcal{H}^{K}$ is a dynamic strategy and $\alpha \in \mathbb{R}_{+}^{m}$.

Definition 4.11 We say that $\mathrm{NA}_{\Phi}^{\mathrm{s}}(\mathcal{P})$ holds if $\mathrm{NA}^{\mathrm{s}}(\mathcal{P})$ holds for the dynamic trading market $(\mathbb{K}, C)$ and $\eta_{T}+\Phi \alpha \in K_{T} \mathcal{P}$-q.s. implies $\alpha=0$.

The (semi-static) superhedging price of $G: \Omega \rightarrow \mathbb{R}^{d}$ is defined as

$$
\pi_{\mathbb{K}, \Phi}(G):=\inf \left\{y \in \mathbb{R}: \exists \bar{\eta} \in \mathcal{H}^{K} \text { such that } y e_{d}+\eta_{T}+\Phi \alpha-G \in K_{T} \text { P-q.s. }\right\},
$$

where $e_{d}$ is the $d$ th vector of the canonical basis of $\mathbb{R}^{d}$. Finally, we define the set $\mathcal{S}_{\Phi}:=\left\{(Z, \mathbb{Q}) \in \mathcal{S}: \mathbb{E}_{\mathbb{Q}}\left[\varphi_{k} \cdot Z_{T}\right] \in\left(b_{k}, a_{k}\right), k=1, \ldots, e\right\}$. 
Theorem 4.12 Assume $\mathrm{NA}_{\Phi}^{\mathrm{s}}(\mathcal{P})$. For any Borel-measurable random vector $G$,

$$
\pi_{\mathbb{K}, \Phi}(G)=\sup _{(Z, \mathbb{Q}) \in \mathcal{S}_{\Phi}} \mathbb{E}_{\mathbb{Q}}\left[G \cdot Z_{T}\right]
$$

Moreover, the superhedging price is attained when $\pi_{\mathbb{K}, \Phi}(G)<\infty$.

As in Sect. 3, we construct an extended space where only dynamic trading in a frictionless asset $\bar{S}$ is allowed. We set $\bar{\Omega}_{0}=\hat{\Omega}_{0} \times \mathbb{R}^{m}$ and $\bar{\Omega}=\bar{\Omega}_{T}$ with $\bar{\Omega}_{t}$ the $(t+1)$-fold product of $\bar{\Omega}_{0}$. We define $\bar{S}_{t}: \bar{\Omega}_{t} \rightarrow \mathbb{R}^{d} \times \mathbb{R}^{m}$ such that

- in the first $d$ components, $\bar{S}_{t}(\omega, \theta, x)=\hat{S}_{t}(\omega, \theta)$, and

- in the last $m$ components, $\bar{S}_{t}(\omega, \theta, x)=x$ for $1 \leq t \leq T-1$, and

$$
\bar{S}_{0}^{k}=p_{k}, \quad \bar{S}_{T}^{k}(\omega, \theta, x)=\phi_{k}(\omega) \cdot \hat{S}_{T}(\omega, \theta), \quad k=d+1, \ldots, m .
$$

The set $\overline{\mathcal{P}}$ of priors is obtained from the collection $\overline{\mathcal{P}}_{t}:=\hat{\mathcal{P}}_{t} \otimes \mathfrak{P}\left(\mathbb{R}^{m}\right)$. The set of constraints in the frictionless market is obtained as $C \times \mathbb{R}_{+}^{m}$. The sets of randomised and consistent strategies in the frictionless market are defined as before and denoted here as $\overline{\mathcal{H}}^{r}$ and $\overline{\mathcal{H}}$, and similarly for the corresponding superhedging prices $\bar{\pi}^{r}$ and $\bar{\pi}$. We then define the semi-static consistent superhedging price as

$$
\begin{aligned}
\bar{\pi}_{\Phi}(g):=\inf \{y \in \mathbb{R}: \exists H & \in \overline{\mathcal{H}} \text { such that } y+(H \bullet \bar{S})_{T} \geq g \overline{\mathcal{P}} \text {-q.s. and } \\
H_{t}^{k} & \left.=H_{1}^{k} \text { for any } k=d+1, \ldots, m, t=1, \ldots, T\right\} .
\end{aligned}
$$

We only need to show the following result.

Lemma $4.13 \bar{\pi}\left(G \cdot \hat{S}_{T}\right)=\bar{\pi}_{\Phi}\left(G \cdot \hat{S}_{T}\right)$.

Proof The inequality " $\leq$ " is clear as any strategy for the right-hand side is also allowed for the left-hand side. For " $\geq$ ", suppose that $(y, \bar{H}) \in \mathbb{R} \times \overline{\mathcal{H}}$ is a superhedge for $G \cdot \hat{S}_{T}$. Let $\bar{H}=(H, h)$, where $H$ is the vector of the first $d$ and $h$ the vector of the last $m$ components. Recall that $\bar{H} \in \overline{\mathcal{H}}$ is consistent, i.e., it only depends on the $\omega$-variable. Let $A_{t}:=\bigcup_{k=d+1}^{m}\left\{\omega \in \Omega_{t}: \bar{H}_{t+1}^{k} \neq \bar{H}_{1}^{k}\right\}$ and let $\bar{t}$ be the first $t \in\{0, \ldots, T-1\}$ such that $\mathbb{P}\left[A_{t}\right]>0$ for some $\mathbb{P} \in \mathcal{P}$. The superhedging property reads

$$
\begin{aligned}
y+(\bar{H} \bullet \bar{S})_{T} & =y+(H \bullet \hat{S})_{T}+\sum_{t=1}^{T-1} \sum_{k=d+1}^{m} h_{t+1}^{k}\left(\bar{S}_{t+1}^{k}-\bar{S}_{t}^{k}\right) \\
& =y+(H \bullet \hat{S})_{T}+\sum_{k=d+1}^{m} h_{1}^{k}\left(\bar{S}_{\bar{t}}^{k}-\bar{S}_{0}^{k}\right)+\sum_{t=\bar{t}}^{T-1} \sum_{k=d+1}^{m} h_{t+1}^{k}\left(\bar{S}_{t+1}^{k}-\bar{S}_{t}^{k}\right) \\
& \geq G \cdot \hat{S}_{T} \quad \overline{\mathcal{P}} \text {-q.s. }
\end{aligned}
$$

Take now a measurable selector $\xi$ of $\left\{x \in \mathbb{R}^{m}: x \cdot h_{\bar{t}+1}<0\right\}$. This exists from [7, Lemmas 12 and 13] as the multifunction corresponds to the interior of the polar 
cone of $h_{\bar{t}+1}$. Since $\mathbb{P}$ is fixed, we may take a Borel-measurable version of $\xi$. Let $\left(P_{t}\right)_{t=0, \ldots, T-1}$ be the kernel decomposition of $\mathbb{P}$, extended arbitrarily to $t=-1$. Fix $x \in \mathbb{R}^{m}$ and an arbitrary selector $\delta_{\theta_{t}}$ of $\delta_{\Theta_{t}}$ from Corollary 3.2 for any $t \in \mathcal{I}$. For any $\lambda>0$, define the probability kernels

$$
\bar{P}_{\bar{t}}:=P_{\bar{t}} \otimes \delta_{\theta_{\bar{t}+1}} \otimes \delta_{\lambda \xi}, \quad P_{t}:=P_{t} \otimes \delta_{\theta_{t+1}} \otimes \delta_{x} \quad \text { for } t \neq \bar{t} .
$$

The measures $\overline{\mathbb{P}}^{\lambda}$ constructed via Fubini's theorem belong to $\overline{\mathcal{P}}$. Since $\lambda$ is arbitrary and $G \cdot \hat{S}_{T}$ depends only on the variable $(\omega, \theta)$, we deduce that $(y, \bar{H})$ cannot be a superhedge.

Proof of Theorem 4.12 From Lemma 4.13 and as in the proof of Theorem 2.4, we have

$$
\pi_{\mathbb{K}, \Phi}(G)=\bar{\pi}_{\Phi}\left(G \cdot \hat{S}_{T}\right)=\bar{\pi}\left(G \cdot \hat{S}_{T}\right)=\bar{\pi}^{r}\left(G \cdot \hat{S}_{T}\right)=\sup _{\overline{\mathbb{Q}} \in \overline{\mathcal{Q}}} \mathbb{E}_{\overline{\mathbb{Q}}}\left[G \cdot \hat{S}_{T}\right],
$$

where the set $\overline{\mathcal{Q}}$ is the analogue for $\bar{S}$ of the set $\hat{\mathcal{Q}}$. To conclude, note that for any $\overline{\mathbb{Q}} \in \overline{\mathcal{Q}}$, we have $\mathbb{E}_{\overline{\mathbb{Q}}}\left[\phi_{k} \cdot \hat{S}_{T}\right]<p_{k}$ for any $k=d+1, \ldots, m$. In particular, this implies $\mathbb{E}_{\overline{\mathbb{Q}}}\left[\varphi_{j} \cdot \hat{S}_{T}\right] \in\left(b_{j}, a_{j}\right)$ for any $j=1, \ldots, e$, and together with Proposition 4.6, the claim follows.

Publisher's Note Springer Nature remains neutral with regard to jurisdictional claims in published maps and institutional affiliations.

Open Access This article is distributed under the terms of the Creative Commons Attribution 4.0 International License (http://creativecommons.org/licenses/by/4.0/), which permits unrestricted use, distribution, and reproduction in any medium, provided you give appropriate credit to the original author(s) and the source, provide a link to the Creative Commons license, and indicate if changes were made.

\section{Appendix: Technical results}

The following result is a simple lemma for convex sets in $\mathbb{R}^{k}$. For $j=1, \ldots, k$, let $e_{j}$ be the $j$ th element of the canonical basis,

$$
A_{j}:=\bigcup_{n \in \mathbb{N}}\left(A+\frac{1}{n} e_{j}\right) \quad \text { and } \quad A_{-j}:=\bigcup_{n \in \mathbb{N}}\left(A-\frac{1}{n} e_{j}\right) .
$$

Lemma A.1 Let $A$ be a convex set in $\mathbb{R}^{k}$ with $\operatorname{int}(A) \neq \emptyset$. Then

$$
\bigcap_{j=1}^{k}\left(A_{j} \cap A_{-j}\right)=\operatorname{int}(A)
$$

Proof " $\supseteq$ ": Let $x \in \operatorname{int} A$. For any $j=1, \ldots, k$, there exists $n_{j}$ with $x+\frac{1}{n_{j}} e_{j} \in A$. Thus, $x \in A_{-j}$. In an analogous way, we can show that $x \in A_{j}$ and the claim follows. 
" $\subseteq$ ": Let $x \notin \operatorname{int} A$. By a change of coordinate, suppose $x=0$. Since $A$ is convex, by the hyperplane separation theorem, there exists $H \in \mathbb{R}^{k} \backslash\{0\}$ such that $H \cdot y \geq 0$ for any $y \in A$. Let $j$ be such that $H^{j} \neq 0$ and suppose that $H^{j}<0$ (the case $H^{j}>0$ follows analogously). From $H^{j} \cdot \frac{1}{n} e_{j}<0$ for any $n \in \mathbb{N}$, we have that $x=0 \notin A_{-j}$. This concludes the proof.

We provide here the proofs of Lemma 2.5 and Proposition 2.6 of Sect. 2.

Lemma A.2 Let $\Omega$ be Polish and $\Phi, \Psi$ multifunctions to $\mathbb{R}^{k}$ with analytic graphs. Then $\Phi+\Psi$ has analytic graph.

Proof Consider for $(\tilde{\omega}, \omega, x, y) \in \Omega \times \Omega \times \mathbb{R}^{k} \times \mathbb{R}^{k}$ the function

$$
f(\omega, \tilde{\omega}, x, y):=(\omega, \tilde{\omega}, x+y) .
$$

Then $f(\operatorname{graph}(\Phi)$, graph $(\Psi))$ is the image of analytic sets under a Borel function and thus analytic. Moreover, the set $\{(\omega, \omega) \in \Omega \times \Omega\}$ is a Borel subset of $\Omega \times \Omega$. To conclude, we observe that $\operatorname{graph}(\Phi+\Psi)$ is the projection on $\Omega \times \mathbb{R}^{k}$ of the analytic set

$$
f\left(\operatorname{graph}\left(K_{t}^{*}\right), \operatorname{graph}\left(C_{t}^{*}\right)\right) \cap\left(\{(\omega, \omega) \in \Omega \times \Omega\} \times \mathbb{R}^{k}\right) .
$$

Proof of Lemma 2.5 By assumption, $K_{t}$ is Borel measurable; thus graph $\left(K_{t}^{*}\right)$ is analytic by [7, Lemma 13]. The result for $t=T$ follows. We proceed by backward induction. Recall that $\operatorname{graph}\left(C_{t}^{*}\right)$ is analytic by assumption, and from the proof of [7, Lemma 6], $\Gamma_{t}$ has analytic graph. From [7, Lemma 12(b)], the same is true for $\overline{\operatorname{conv}\left(\Gamma_{t}\right)}$. We conclude by using Lemma A.2 and [7, Lemma 12(d)].

Proof of Proposition 2.6 Define the market $\mathbb{K}^{t}:=\left\{K_{0}, \ldots, K_{t-1}, \tilde{K}_{t}, \ldots, \tilde{K}_{T}\right\}$ for $t \in \mathcal{I}$. We proceed by backward induction. For $t=T$, we have $\mathbb{K}^{T}=\mathbb{K}$ and the two properties follow by assumption. Suppose that the thesis is true for $u=t+1, \ldots, T$; we show that it is true for $t$. For $\omega \in \Omega_{t}$, let

$$
\Lambda_{t}(\omega):=\left\{x \in \mathbb{R}^{d}: x \in \tilde{K}_{t+1}(\omega ; \cdot) \mathcal{P}_{t} \text {-q.s. }\right\} .
$$

From [7, Lemma 7], we have $\Lambda_{t}=\Gamma_{t}^{*}$, implying $\Lambda_{t}^{*} \supseteq \tilde{K}_{t+1}^{*} \mathcal{P}$-q.s. From the induction hypothesis and Assumption 2.1, we have $\operatorname{int}\left(\Lambda_{t}^{*}\right) \neq \emptyset$ and $\operatorname{int}\left(K_{t}^{*}-C_{t}^{*}\right) \neq \varnothing$ outside a $\mathcal{P}$-polar set $N$. Note now that for every $\omega \in N^{c}$ such that

$$
\operatorname{int}\left(\Lambda_{t}^{*}\right)(\omega) \cap \operatorname{int}\left(K_{t}^{*}-C_{t}^{*}\right)(\omega)=\emptyset,
$$

we can find $x \in \mathbb{R}^{d} \backslash\{0\}$ such that $x \cdot y \leq 0$ for any $y \in K_{t}^{*}-C_{t}^{*}$ and $x \cdot z \geq 0$ for any $z \in \Lambda_{t}^{*}$. From [7, Lemma 16] and since $K_{t}$ and $C_{t}$ are closed sets, we deduce that $x \in-K_{t} \cap C_{t}$ and $x \in \Lambda_{t}$. Let $\eta$ be an $\mathcal{F}_{t}^{u}$-measurable selector of $\left\{\left(-K_{t} \cap C_{t} \cap \Lambda_{t}\right) \backslash\{0\}\right\}$ (for its existence, see [7, Proposition 4]). Note that $\eta \in A_{t+1}\left(\mathbb{K}^{t+1}\right)$ and by the definition of $\Lambda_{t}$, we have $\eta \in \tilde{K}_{t+1} \mathcal{P}$-q.s. The strict no arbitrage condition implies that $\eta=0 \mathcal{P}$-q.s., and as a consequence, the set of all $\omega \in N^{c}$ such that (A.1) is satisfied is $\mathcal{P}$-polar. On the complement, we clearly have $\operatorname{int}\left(\tilde{K}_{t}^{*}\right) \neq \emptyset$. We are only left to show that $\mathrm{NA}^{\mathrm{s}}(\mathcal{P})$ holds for $\mathbb{K}^{t}$. 
Let $\eta:=\left(\eta_{0}, \ldots, \eta_{T}\right)$ and $r \geq t$ be such that $\eta_{r} \in A_{r}\left(\mathbb{K}^{t}\right) \cap \mathcal{L}^{0}\left(\mathcal{F}_{r}^{u} ; \tilde{K}_{r}\right)$ (the case $r \leq t-1$ is trivial). By admissibility,

$$
\eta_{r}=-k_{0}-\cdots-k_{t-1}-\tilde{k}_{t}-\cdots-\tilde{k}_{r}
$$

with $k_{s} \in K_{s}$ for $s=0, \ldots, t-1$ and $\tilde{k}_{s} \in \tilde{K}_{s}$ for $s=t, \ldots, r$. Note that for $t \leq s \leq T$, we have $\operatorname{int}\left(\tilde{K}_{s}^{*}\right) \neq \varnothing \mathcal{P}$-q.s. Therefore

$$
\tilde{K}_{s}=\left(K_{s}^{*} \cap\left(\overline{\operatorname{conv}\left(\Gamma_{s}\right)}+C_{s}^{*}\right)\right)^{*}=K_{s}+\left(\Lambda_{s} \cap C_{s}\right),
$$

where the first equality follows by (2.1) and the second from [7, Lemma 16]. We start with the case $r \geq t+1$. From [7, Lemma 8], we have $\tilde{k}_{t}=k_{t}+\lambda_{t}$ for some $k_{t} \in \mathcal{L}^{0}\left(\mathcal{F}_{t}^{u} ; K_{t}\right)$ and $\bar{\lambda}_{t} \in \mathcal{L}^{0}\left(\mathcal{F}_{t}^{u} ; \tilde{K}_{t+1} \cap C_{t}\right)$. We can therefore rewrite

$$
\eta_{r}=-k_{0}-\cdots-k_{t-1}-\left(k_{t}+\lambda_{t}\right)-\tilde{k}_{t+1}-\cdots-\tilde{k}_{r}
$$

Define the new strategy $\tilde{\eta}$ with $\tilde{\eta}_{s}=\eta_{s}$ for any $s \neq t$ and $\tilde{\eta}_{t}:=\eta_{t-1}-k_{t}=\eta_{t}+\lambda_{t}$. Since $\eta_{t}, \lambda_{t+1}$ are in $\mathcal{L}^{0}\left(\mathcal{F}_{t}^{u} ; C_{t}\right)$ and $C_{t}$ is a convex cone, the sum takes also values in $C_{t}$, from which we get $\tilde{\eta}_{t} \in A_{t}\left(\mathbb{K}^{t+1}\right)$. In particular, $\tilde{\eta}$ is admissible for the market $\mathbb{K}^{t+1}$ and satisfies $\tilde{\eta}_{r} \in A_{r}\left(\mathbb{K}^{t+1}\right) \cap \mathcal{L}^{0}\left(\mathcal{F}_{r}^{u} ; \tilde{K}_{r}\right)$. From the induction hypothesis of strict no arbitrage, it follows that $\tilde{\eta}_{r}=\eta_{r}=0 \mathcal{P}$-q.s.

For the case $r=t$, we have $\eta_{t} \in \mathcal{L}^{0}\left(\mathcal{F}_{r}^{u} ; \tilde{K}_{t}\right)$ by assumption. Similarly as above, we can rewrite $\eta_{t}=\xi_{t}+\lambda_{t}$ for some $\xi_{t} \in \mathcal{L}^{0}\left(\mathcal{F}_{t}^{u} ; K_{t}\right)$ and $\lambda_{t} \in \mathcal{L}^{0}\left(\mathcal{F}_{t}^{u} ; \tilde{K}_{t+1} \cap C_{t}\right)$, and hence $\lambda_{t}=-k_{0}-\cdots-k_{t-1}-\xi_{t}$. This implies

$$
\lambda_{t} \in A_{t}\left(\mathbb{K}^{t+1}\right) \cap \mathcal{L}^{0}\left(\mathcal{F}_{t}^{u} ; \tilde{K}_{t+1}\right) \subseteq A_{t+1}\left(\mathbb{K}^{t+1}\right) \cap \mathcal{L}^{0}\left(\mathcal{F}_{t+1}^{u} ; \tilde{K}_{t+1}\right),
$$

where the inclusion follow from Assumption 2.1. The strict no arbitrage condition implies $\lambda_{t}=0 \mathcal{P}$-q.s. and therefore $\eta_{t}=\xi_{t} \mathcal{P}$-q.s. Thus $\eta_{t} \in \mathcal{A}\left(\mathbb{K}^{t+1}\right) \cap \mathcal{L}^{0}\left(\mathcal{F}_{t}^{u} ; K_{t}\right)$. Using again the strict no arbitrage condition, it follows that $\eta_{t}=0$.

\section{References}

1. Acciaio, B., Beiglböck, M., Penkner, F., Schachermayer, W.: A model-free version of the fundamental theorem of asset pricing and the super-replication theorem. Math. Finance 26, 233-251 (2016)

2. Aksamit, A., Deng, S., Obłój, J., Tan, X.: The robust pricing-hedging duality for American options in discrete time financial markets. Math. Finance 29, 861-897 (2019)

3. Aliprantis, C.D., Border, K.C.: Infinite Dimensional Analysis: A Hitchhiker's Guide. Springer, Berlin (2006)

4. Avellaneda, M., Lévy, A., Paras, A.: Pricing and hedging derivative securities in markets with uncertain volatilities. Appl. Math. Finance 2, 73-88 (1995)

5. Bartl, D., Cheridito, P., Kupper, M., Tangpi, L.: Duality for increasing convex functionals with countably many marginal constraints. Banach J. Math. Anal. 11, 72-89 (2017)

6. Bayraktar, E., Munk, A.: High-roller impact: a large generalized game model of parimutuel wagering. Mark. Microstruct. Liq. 03, 1750006 (2017)

7. Bayraktar, E., Zhang, Y.: Fundamental theorem of asset pricing under transaction costs and model uncertainty. Math. Oper. Res. 41, 1039-1054 (2016)

8. Bayraktar, E., Zhou, Z.: On arbitrage and duality under model uncertainty and portfolio constraints. Math. Finance 27, 988-1012 (2017) 
9. Bayraktar, E., Zhou, Z.: No-arbitrage and hedging with liquid American options. Math. Oper. Res. 44, 468-486 (2019)

10. Bertsekas, D.P., Shreve, S.E.: Stochastic Optimal Control: The Discrete Time Case. Academic Press, New York (1978)

11. Bouchard, B., Deng, S., Tan, X.: Superreplication with proportional transaction cost under model uncertainty. Math. Finance 29, 837-860 (2019)

12. Bouchard, B., Nutz, M.: Arbitrage and duality in nondominated discrete-time models. Ann. Appl. Probab. 25, 823-859 (2015)

13. Bouchard, B., Nutz, M.: Consistent price systems under model uncertainty. Finance Stoch. 20, 83-98 (2016)

14. Burzoni, M.: Arbitrage and hedging in model-independent markets with frictions. SIAM J. Financ. Math. 7, 812-844 (2016)

15. Burzoni, M., Frittelli, M., Hou, Z., Maggis, M., Obłój, J.: Pointwise arbitrage pricing theory in discrete time. Math. Oper. Res. 44, 1034-1057 (2019)

16. Burzoni, M., Šikić, M.: Robust martingale selection problem and its connections to the no-arbitrage theory. Math. Finance (2019). Forthcoming, available online at https://doi.org/10.1111/mafi.12225

17. Cheridito, P., Kupper, M., Tangpi, L.: Duality formulas for robust pricing and hedging in discrete time. SIAM J. Financ. Math. 8, 738-765 (2017)

18. Deng, S., Tan, X., Yang, X.: Utility maximization with proportional transaction costs under model uncertainty. Math. Oper. Res. (2019). To appear, available online at arXiv: 1805.06498

19. Dolinsky, Y., Soner, H.M.: Robust hedging with proportional transaction costs. Finance Stoch. 18, 327-347 (2014)

20. Kabanov, Yu.M.: Hedging and liquidation under transaction costs in currency markets. Finance Stoch. 3, 237-248 (1999)

21. Kabanov, Yu.M., Rásonyi, M., Stricker, C.: On the closedness of sums of convex cones in $L^{0}$ and the robust no-arbitrage property. Finance Stoch. 7, 403-411 (2003)

22. Knight, F.: Risk, Uncertainty and Profit. Houghton Mifflin, Boston (1921)

23. Kühn, C., Molitor, A.: Prospective strict no-arbitrage and the fundamental theorem of asset pricing under transaction costs. Finance Stoch. 23, 1049-1077 (2019)

24. Rockafellar, R.T., Wets, R.J.-B.: Variational Analysis. Springer, Berlin (1998)

25. Terkelsen, F.: Some minimax theorems. Math. Scand. 31, 405-413 (1972) 